\title{
Bounded Independence versus Symmetric Tests
}

\author{
RAVI BOPPANA, Massachusetts Institute of Technology \\ JOHAN HÅSTAD, KTH-Royal Institute of Technology \\ CHIN HO LEE, Northeastern University \\ EMANUELE VIOLA, Northeastern University
}

For a test $T \subseteq\{0,1\}^{n}$, define $k^{*}(T)$ to be the maximum $k$ such that there exists a $k$-wise uniform distribution over $\{0,1\}^{n}$ whose support is a subset of $T$.

For $H_{t}=\left\{x \in\{0,1\}^{n}:\left|\sum_{i} x_{i}-n / 2\right| \leq t\right\}$, we prove $k^{*}\left(H_{t}\right)=\Theta\left(t^{2} / n+1\right)$.

For $S_{m, c}=\left\{x \in\{0,1\}^{n}: \sum_{i} x_{i} \equiv c(\bmod m)\right\}$, we prove that $k^{*}\left(S_{m, c}\right)=\Theta\left(n / m^{2}\right)$. For some $k=O(n / m)$ we also show that any $k$-wise uniform distribution puts probability mass at most $1 / m+1 / 100$ over $S_{m, c}$. Finally, for any fixed odd $m$ we show that there is an integer $k=(1-\Omega(1)) n$ such that any $k$-wise uniform distribution lands in $T$ with probability exponentially close to $\left|S_{m, c}\right| / 2^{n}$; and this result is false for any even $m$.

\section{CCS Concepts: • Theory of computation $\rightarrow$ Pseudorandomness and derandomization;}

Additional Key Words and Phrases: Pseudorandomness, bounded independence, thresholds, modulus, symmetric functions

\section{ACM Reference format:}

Ravi Boppana, Johan Håstad, Chin Ho Lee, and Emanuele Viola. 2019. Bounded Independence versus Symmetric Tests. ACM Trans. Comput. Theory 11, 4, Article 21 (July 2019), 27 pages.

https://doi.org/10.1145/3337783

\section{INTRODUCTION AND OUR RESULTS}

A distribution on $\{0,1\}^{n}$ is $k$-wise uniform, or $k$-wise independent, if any $k$ bits are uniform in $\{0,1\}^{k}$. The study of $k$-wise uniformity has been central to theoretical computer science since at least the seminal work [8] by Carter and Wegman. A specific direction has been to show that $k$-wise uniformity "looks random" to several classes of tests. These classes include combinatorial rectangles $[9,14]$ (for an exposition see, e.g., Lecture 1 in Reference [32]), bounded-depth circuits, or $\mathrm{AC}^{0}[3,6,20,28,31]$ (see, e.g., Lectures 2 and 3 in Reference [32]), and halfspaces [12, 13, 17, 26]. More recently, a series of works consider smoothed versions of the first two classes, where the input is perturbed with noise and gives improved bounds $[1,16,19,22]$. These results have in turn

A preliminary version of this paper appeared in RANDOM 2016 [5].

Johan Håstad is supported by the Swedish Research Council. Chin Ho Lee and Emanuele Viola are supported by National Science Foundation grant CCF-1319206. This work is done in part while C.H.L. and E.V. were visiting Harvard University, with support from Salil Vadhan's Simons Investigator grant, and in part while J.H., C.H.L., and E.V. were at the Simons Institute for the Theory of Computing.

Authors' addresses: C. H. Lee and E. Viola, Khoury College of Computer Sciences, Northeastern University, 440 Huntington Avenue, 202 West Village H, Boston, MA 02115; J. Håstad, Mathematics KTH, SE-100 44 Stockholm, Sweden; R. Boppana, Department of Mathematics, MIT, Cambridge, MA 02142.

Permission to make digital or hard copies of all or part of this work for personal or classroom use is granted without fee provided that copies are not made or distributed for profit or commercial advantage and that copies bear this notice and the full citation on the first page. Copyrights for components of this work owned by others than the author(s) must be honored. Abstracting with credit is permitted. To copy otherwise, or republish, to post on servers or to redistribute to lists, requires prior specific permission and/or a fee. Request permissions from permissions@acm.org.

(C) 2019 Copyright held by the owner/author(s). Publication rights licensed to ACM.

1942-3454/2019/07-ART21 \$15.00

https://doi.org/10.1145/3337783 
found many applications. For example, the recent exciting constructions of 2-source extractors for polylogarithmic min-entropy $[4,10]$ rely on the results that $k$-wise uniformity looks random to $\mathrm{AC}^{0}[6]$ and the majority function [12].

In this work, we extend this direction by giving new bounds for two classes of tests, both symmetric. First, we consider the class of $\bmod m$ tests.

Definition 1. For an input length $n$, and integers $m$ and $c$, we define the set $S_{m, c}:=\left\{x \in\{0,1\}^{n}\right.$ : $\left.\sum_{i} x_{i} \equiv c(\bmod m)\right\}$.

These tests have been extensively studied at least since circuit complexity theory "hit the wall" of circuits with mod $m$ gates for composite $m$ in the 1980s. However, the effect of $k$-wise uniformity on mod $m$ tests does not seem to have been known before this article. We study for what values of $k$ there exist a $k$-wise uniform distribution over $\{0,1\}^{n}$ supported on $S_{m, c}$. Note that such $k$-wise uniform distributions are obviously not pseudorandom against $\bmod m$ tests. Our first main result gives tight bounds on the maximum value of such a $k$, establishing $k$ is about $n / m^{2}$.

Theorem 2. For all integers $m \geq 2$ and $c$, there exists an integer $k \geq n /\left(32 m^{2}\right)-1$ and a $k$-wise uniform distribution on $\{0,1\}^{n}$ that is supported on $S_{m, c}$.

Theorem 3. For all integers $m \geq 2, c$, and $k \geq 140 \mathrm{n} / \mathrm{m}^{2}+4$, no $k$-wise uniform distribution on $\{0,1\}^{n}$ can be supported on $S_{m, c}$.

Theorem 2 is trivial for $m=2$, as the uniform distribution over $S_{2,0}$ is $(n-1)$-wise uniform. But already for $m=3$ the result is not trivial.

Theorem 3 is equivalent to saying that when $k \geq 140 n / m^{2}+4$, then every $k$-wise uniform distribution must land in $\overline{S_{m, c}}$ with non-zero probability.

For motivation, recall from above the line of works [3, 6, 20, 28, 31] showing that $k$-wise uniform distributions fool $\mathrm{AC}^{0}$ circuits. Specifically, these works show $k=$ poly $\log n$ suffices to fool $\mathrm{AC}^{0}$ circuits on $n$ bits of size $\operatorname{poly}(n)$ and depth $O(1)$. It is natural to ask whether the same distribution also fools $\mathrm{AC}^{0}$ circuits with mod $m$ gates, a "frontier" class for which we have exponential lower bounds [27, 30] (when $m$ is prime) but not good pseudorandom generators. A positive answer might have looked plausible, given that, for example, the parity function is hard even with mod 3 gates [30]. But, in fact, Theorem 2 gives a strong negative answer, showing that $k=\Omega(n)$ is necessary even for a single mod 3 gate.

Theorem 2 proves a conjecture in Reference [23] where this question is also raised. Their motivation was a study of the "mod 3" rank of $k$-wise uniform distributions, started in Reference [24], which is the dimension of the space spanned by the support of the distribution over $\mathbb{F}_{3}$. The work of Lee and Viola [23, Claim 1.10] shows that achieving $100 \log n$-wise uniformity with dimension $\leq n^{0.49}$ would have applications to pseudorandomness. It also exhibits a distribution with dimension $n^{0.72}$ and pairwise uniformity. Theorem 2 yields a new piece of information in this study: There exist distributions with dimension $n-1$ and $\Omega(n)$-wise uniformity. However, we are still far from achieving the parameters required in Reference [23, Claim 1.10].

We then prove another theorem that is in the same spirit of Theorem 3 but gives different information.

THEOREM 4. Let $m$ and $c$ be two integers.

(a) For $k \geq 2 n / m+2$, no $k$-wise uniform distribution on $\{0,1\}^{n}$ can be supported on $S_{m, c}$.

(b) If $m$ is odd, then there is a $\gamma>0$ depending only on $m$ such that for any $(1-\gamma) n$-wise uniform distribution $D$ on $\{0,1\}^{n}$, we have $\left|\operatorname{Pr}\left[D \in S_{m, c}\right]-\right| S_{m, c}\left|/ 2^{n}\right| \leq 2^{-\gamma n}$.

(c) There exists a universal constant $C$ such that for every $\varepsilon>0, n \geq C m^{2} \log m$, and any $C(n / m)(1 / \varepsilon)^{2}$-wise uniform distribution $D$ on $\{0,1\}^{n}, \operatorname{Pr}\left[D \in S_{m, c}\right] \leq\left|S_{m, c}\right| / 2^{n}+\varepsilon$. 
First, Theorem 4(a) shows that the largest possible value of $k$ in Theorem 2 is $k \leq 2(n+1) / m+2$. Compared to Theorem 3 , this result is asymptotically inferior but gives better constants and has a simpler proof. Theorem 4 (b) shows that when $m$ is odd, if $k$ is larger than $(1-\gamma) n$ for a positive constant $\gamma$ depending only on $m$ then $k$-wise uniformity fools $S_{m, c}$ with exponentially small error. The proof of Theorem 4(b), however, does not carry to the setting of $k<n / 2$, for any $m$. So we establish Theorem 4(c), which gives a worse error bound but allows for $k$ to become smaller for larger $m$, specifically, $k=O(n / m)$ for constant error. The error bound in Theorem 4(c) and the density of $S_{m, c}$ are such that it only provides a meaningful upper bound on the probability that the $k$-wise uniform distribution lands in $S_{m, c}$ but not a lower bound. In fact, we conjecture that no lower bound is possible in the sense that there is a constant $C>0$ such that for every $m$ there is a $C n$-wise uniform distribution supported on the complement of $S_{m, c}$.

We note that Theorem $4(\mathrm{~b})$ is false when $m$ is even, because the uniform distribution on $S_{2,0}$ has uniformity $k=n-1$ but puts about $2 / m$ mass on $S_{m, 0}$, a set that as we shall see later (cf. Remark 1) has density about $1 / \mathrm{m}$. The latter density bound, in combination with Theorem $4(\mathrm{~b})$ and Theorem 4(c), implies that for some $k=\min \{O(n / m),(1-\Omega(1)) n\}$, every $k$-wise uniform distribution puts probability mass at most $1 / m+1 / 100$ over $S_{m, c}$ for odd $m$ and any integer $c$.

A remaining open problem is to understand for what values of $k$ does $k$-wise uniformity ensure closeness to uniform modulo $m$. Note that to get close to uniform, or even to put probability mass on all possible moduli, one would want $\varepsilon$ in Theorem 4(c) to be roughly $1 / \mathrm{m}$. However, this gives $k \geq n$ in the theorem, which is trivial.

We then consider another class of tests that can be written as the intersection of two half-spaces.

Definition 5. For an input length $n$, and an integer $t$, we define the set $H_{t}:=\left\{x \in\{0,1\}^{n}\right.$ : $\left.\left|\sum_{i} x_{i}-n / 2\right| \leq t\right\}$.

Again, we ask for what values of $k$ there exists a $k$-wise uniform distribution over $\{0,1\}^{n}$ supported on $H_{t}$. We obtain tight bounds for $k$ up to a constant factor, showing that the maximum value of such a $k$ is $\Theta\left(t^{2} / n+1\right)$.

THEOREM 6. For every integer $t$, there exists an integer $k \geq t^{2} /(50 n)$ and a $k$-wise uniform distribution over $\{0,1\}^{n}$ that is supported on $H_{t}$.

TheOREm 7. For all integers $t$ and $k \geq 36 t^{2} / n+3$, no $k$-wise uniform distribution over $\{0,1\}^{n}$ can be supported on $H_{t}$.

In fact, as we will see in the proof, the distribution in Theorem 6 is actually supported on $H_{t} \cap$ $S_{m, 0}$.

One motivation for these results is to understand for which tests the smoothed version of the test obtained by perturbing coordinates with random noise is fooled by $k$-wise uniformity. As mentioned earlier, this understanding underlies recent, state-of-the-art pseudorandom generators $[1,16,19,22]$. See also Reference [23]. Using Theorem 6 we prove that $\Omega(\log n)$-wise uniformity is necessary to fool read-once DNF on $n$ bits, even with constant noise. Note that $O(\log n)$-uniformity is sufficient, even without noise [14].

THEOREM 8. There exists a read-once DNF $d:\{0,1\}^{n} \rightarrow\{0,1\}$, a constant $\alpha$, and an $\alpha \log n$-wise uniform distribution $D$ such that $\left|\operatorname{Pr}[d(U)=1]-\operatorname{Pr}\left[d\left(D+N_{\alpha}\right)=1\right]\right| \geq \Omega(1)$, where $U$ is uniform over $\{0,1\}^{n}, N_{\alpha}$ is the distribution over $\{0,1\}^{n}$ whose bits are independent and are set to uniform with probability $\alpha$ and 0 otherwise, and " $"$ " is bit-wise XOR.

Proof. Let $d$ be the Tribes DNF with width $w=\log n-\log \ln n+o_{n}(1)$, see, e.g., Reference [25]. We have $|\operatorname{Pr}[d(U)=1]-1 / 2|=o(1)$. Partition the $n$ bits into $n / w$ blocks of size $w$. The distribution $D$ has i.i.d. blocks. The projection of each block is an $\alpha w$-wise uniform distribution with Hamming 
weight $\leq 2 w / 3$. The probability that $d\left(D+N_{\alpha}\right)=1$ is at most the probability that there exists a block where the noise vector $N_{\alpha}$ has Hamming weight $\geq w / 3$. This probability is at most

$$
(n / w) 2^{w}(\alpha / 2)^{w / 3} \leq 1 / 3
$$

for a sufficiently small $\alpha$.

\subsection{Techniques}

We give two related approaches to proving Theorem 2. At a high level, both approaches are similar to the work of Alon et al. [2], which shows that one can apply a small perturbation to the probability masses of every almost $k$-wise uniform distribution on $\{0,1\}^{n}$ to make it $k$-wise uniform, showing that every $\varepsilon$-almost $k$-wise uniform distribution on $\{0,1\}^{n}$ is $n^{O(k)} \varepsilon$-close to a $k$-wise uniform distribution, in statistical distance. However, in their setting there is no constraint on the support. This makes our proof significantly more technical.

Our first approach uses the following equivalent definition for a distribution on $\{0,1\}^{n}$ to be $k$-wise uniform: the distribution is unbiased under any parity test on at most $k$ bits. To construct our distribution, we first start with the uniform distribution over the set $S_{m, c}$, and show that the bias under each of these parity tests is small enough, so that they can be made zero by a small perturbation of the probability masses of the distribution. Our goal is then to show that the change in the probability on each weight is no more than the probability we start with, so that it remains non-negative after the perturbation. In the conference version of this article [5], we use this approach to prove a slightly weaker version of Theorem 2 . We refer the interested readers to Reference [5] for details.

We now give an overview of the second approach, which is developed in this article. Instead of looking at the biases of parity tests, we consider another equivalent characterization of $k$-wise uniform distributions that are symmetric. To simplify the calculations, we will switch to $\{-1,1\}$ and consider distributions supported on $S_{m, c}^{\prime}:=\left\{y \in\{-1,1\}^{n}: \sum_{i} y_{i} \equiv c(\bmod m)\right\}$. One can then translate results for $\{-1,1\}^{n}$ back to $\{0,1\}^{n}$. (See Fact 12.) A symmetric distribution is $k$-wise uniform on $\{-1,1\}^{n}$ if and only if the first $k$ moments of the sum of its $n$ bits match the corresponding moments of the uniform bits. Similar to the first approach, we start with the uniform distribution on $S_{m, c}^{\prime}$, and show that the first $k$ moments of the sum of the bits are close to the ones of the uniform bits. Then, we perturb the probabilities on $k+1$ of the sums $\sum_{i} y_{i}$ of the distribution to match these moments exactly. Once again, our goal is to show that the amount of correction is small enough so that the adjusted probabilities remain non-negative. Note that in this approach we work with distributions over the integers instead of $\{0,1\}^{n}$.

While the two approaches seem similar to each other, the latter allows us to perform a more refined analysis on the tests we consider in this article and obtain the tight lower bounds for both modular and threshold tests.

Organization. We begin with Theorem 4 in Section 2, because it is simpler. We use the second approach to prove the tight lower bounds for $S_{m, c}$ (Theorem 2) and $H_{t}$ (Theorem 6) in Sections 3 and 4, respectively. The proof of Theorem 2 involves a somewhat technical lemma that we defer to Section 5. Finally, we prove our tight upper bounds for $S_{m, c}$ (Theorem 3) and $H_{t}$ (Theorem 7) in Sections 6 and 7, respectively.

\section{PROOF OF THEOREM 4}

In this section, we prove Theorem 4 . We start with the following theorem that will give Theorem 4(a) as a corollary. 
Theorem 9. Let $I \subseteq\{0,1, \ldots, n\}$ be a subset of size $|I| \leq n / 2$. There does not exist a $2|I|$-wise uniform distribution on $\{0,1\}^{n}$ that is supported on $S:=\left\{x \in\{0,1\}^{n}: \sum_{i} x_{i} \in I\right\}$.

Proof. Suppose there exists such a distribution $D$. Define the $n$-variate nonzero real polynomial $p$ by

$$
p(x):=\prod_{i \in I}\left(-i+\sum_{j=1}^{n} x_{j}\right) .
$$

Note that $p(x)=0$ when $x \in S$. And so $\mathbb{E}\left[p^{2}(D)\right]=0$ in particular. However, since $p^{2}$ has degree at most $2|I|$, we have $\mathbb{E}\left[p^{2}(D)\right]=\mathbb{E}\left[p^{2}(U)\right]>0$, where $U$ is the uniform distribution over $\{0,1\}^{n}$, a contradiction.

Proof of Theorem 4(A). When $I$ corresponds to the $\bmod m$ test $S_{m, c},|I| \leq n / m+1$.

We now move to Theorem 4(b). First, we prove a lemma giving a useful estimate of

$$
\sum_{x \in S_{m, c}}(-1)^{\sum_{i=1}^{k} x_{i}} .
$$

Similar bounds have been established elsewhere, cf. e.g., Theorem 2.9 in Reference [33], but we do not know of a reference with an explicit dependence on $m$, which will be used in the next section. Theorem 4(b) follows from bounding above the tail of the Fourier coefficients of the indicator function of $S_{m, c}$.

Lemma 10. For any $1 \leq k \leq n-1$ and any $0 \leq c \leq m-1$, we have

$$
\left|\sum_{x \in S_{m, c}}(-1)^{\sum_{i=1}^{k} x_{i}}\right| \leq 2^{n}\left(\cos \frac{\pi}{2 m}\right)^{n},
$$

while for $k=0$, we have

$$
|| S_{m, c}\left|-2^{n} / m\right| \leq 2^{n}\left(\cos \frac{\pi}{2 m}\right)^{n} .
$$

For odd $m$ the first bound also holds for $k=n$.

Proof. Consider an expansion of

$$
p(y)=(1-y)^{k}(1+y)^{n-k}
$$

into $2^{n}$ terms indexed by $x \in\{0,1\}^{n}$, where $x_{i}=0$ indicates that we take the term 1 from the $i$ th factor. It is easy to see that the coefficient of $y^{d}$ is $\sum_{|x|=d}(-1)^{\sum_{i=1}^{k} x_{i}}$, where $|x|$ denotes the number of occurrences of 1 in $x$. Denote $\zeta:=e^{2 \pi i / m}$ as an $m$ th root of unity. Recall the identity

$$
\frac{1}{m} \sum_{j=0}^{m-1} \zeta^{j(d-c)}= \begin{cases}1 & \text { if } d \equiv c \quad(\bmod m) \\ 0 & \text { otherwise }\end{cases}
$$

Thus the sum we want to bound is equal to

$$
\frac{1}{m} \sum_{j=0}^{m-1} \zeta^{-j c} p\left(\zeta^{j}\right)
$$

Note that $p\left(\zeta^{0}\right)=p(1)=0$ for $k \neq 0$ while for $k=0, p\left(\zeta^{0}\right)=2^{n}$. For the other terms we have the following bound.

Claim 11. For $1 \leq j \leq m-1,\left|p\left(\zeta^{j}\right)\right| \leq 2^{n}\left(\cos \frac{\pi}{2 m}\right)^{k}\left(\cos \frac{\pi}{m}\right)^{n-k}$. 
Proof. As $\left|1+e^{i \theta}\right|=2|\cos (\theta / 2)|$ and $\left|1-e^{i \theta}\right|=2|\sin (\theta / 2)|$ we have

$$
\begin{aligned}
\left|p\left(\zeta^{j}\right)\right| & =\left|1-\zeta^{j}\right|^{k}\left|1+\zeta^{j}\right|^{n-k} \\
& =2^{n}\left|\sin \frac{j \pi}{m}\right|^{k}\left|\cos \frac{j \pi}{m}\right|^{n-k} \\
& \leq 2^{n}\left(\cos \frac{\pi}{2 m}\right)^{k}\left(\cos \frac{\pi}{m}\right)^{n-k},
\end{aligned}
$$

where the last inequality holds for odd $m$, because (1) $\sin \frac{j \pi}{m}$ is largest when $j=\frac{m-1}{2}$ or $j=\frac{m+1}{2}$, (2) $\sin \left(\frac{\pi}{2}-x\right)=\cos x$, and (3) $\cos \frac{j \pi}{m}$ is largest when $j=1$ or $j=m-1$. For even $m$ the term with $j=m / 2$ is 0 , as in this case we are assuming that $k<n$, and the bounds for odd $m$ are valid for the other terms.

Therefore, for $k \neq 0$ we have

$$
\left|\sum_{x \in S_{m, c}}(-1)^{\sum_{i=1}^{k} x_{i}}\right|=\frac{m-1}{m} \cdot 2^{n}\left(\cos \frac{\pi}{2 m}\right)^{k}\left(\cos \frac{\pi}{m}\right)^{n-k} \leq 2^{n}\left(\cos \frac{\pi}{2 m}\right)^{k}\left(\cos \frac{\pi}{m}\right)^{n-k},
$$

and we complete the proof using the fact that $\cos (\pi / m) \leq \cos (\pi / 2 m)$. For $k=0$ we also need to include the term $p(1)=2^{n}$, which divided by $m$ gives the term $2^{n} / m$.

Remark 1. Clearly the lemma for $k=0$ simply is the well-known fact that the cardinality of $S_{m, c}$ is very close to $2^{n} / m$. Equivalently, if $x$ is uniform in $\{0,1\}^{n}$, then the probability that $\sum_{i} x_{i} \equiv c$ $(\bmod m)$ is very close to $1 / m$.

Proof of Theorem 4(в). We use Fourier analysis (see, e.g., Reference [25] for an introduction). Let $f:\{0,1\}^{n} \rightarrow\{0,1\}$ be the characteristic function of $S_{m, c}$. We write $f$ in its Fourier expansion $f(x)=\sum_{\beta \in\{0,1\}^{n}} \hat{f}_{\beta}(-1)^{\sum_{i=1}^{n} \beta_{i} x_{i}}$. We first bound above the nonzero Fourier coefficients of $f$. Since $f$ is symmetric, by Lemma 10 , we have for any $\beta$ with $|\beta|=k>0$,

$$
\left|\hat{f}_{\beta}\right|=\left|2^{-n} \sum_{x \in S_{m, c}}(-1)^{\sum_{i=1}^{k} x_{i}}\right| \leq\left(\cos \frac{\pi}{2 m}\right)^{n} \leq 2^{-\alpha n}
$$

where $\alpha=-\log _{2} \cos (\pi / 2 m)=\Theta\left(1 / m^{2}\right)$ depends only on $m$. If $D$ is $k$-wise uniform, then we have $\mathbb{E}_{x \sim D}\left[(-1)^{\sum \beta_{i} x_{i}}\right]=0$ whenever $0<|\beta| \leq d$. Therefore,

$$
|\mathbb{E}[f(D)]-\mathbb{E}[f(U)]| \leq \sum_{|\beta|>k}\left|\hat{f}_{\beta}\right| \cdot\left|\mathbb{E}_{x \sim D}\left[(-1)^{\sum \beta_{i} x_{i}}\right]\right| \leq \sum_{|\beta|>k}\left|\hat{f}_{\beta}\right| \leq 2^{-\alpha n} \sum_{t=k+1}^{n}\left(\begin{array}{l}
n \\
t
\end{array}\right)=2^{-\alpha n} \sum_{t=0}^{n-k-1}\left(\begin{array}{l}
n \\
t
\end{array}\right) .
$$

For $k \geq(1-\delta) n$, we have an upper bound of $2^{n(H(\delta)-\alpha)}$. Pick $\delta$ small enough so that $H(\delta) \leq \alpha / 2$. The result follows by setting $\gamma:=\min \{\alpha / 2, \delta\}$.

Note that the above proof fails when $m$ is even as we cannot handle the term with $|\beta|=n$. Finally, we prove Theorem 4(c). We use approximation theory.

Proof of Theorem 4(c). We will show that $\operatorname{Pr}\left[D \in S_{m, c}\right] \leq \varepsilon$. Let $f:\{0,1\}^{n} \rightarrow\{0,1\}$ be the characteristic function of $S_{m, c}$. The proof amounts to exhibiting a real polynomial $p$ in $n$ variables of degree $d=C(n / m)(1 / \varepsilon)^{2}$ such that $f(x) \leq p(x)$ for every $x \in\{0,1\}^{n}$, and $\mathbb{E}[p(U)] \leq \varepsilon$ for $U$ uniform over $\{0,1\}^{n}$. To see that this suffices, note that $\mathbb{E}[p(U)]=\mathbb{E}[p(D)]$ for any distribution $D$ that is $d$-wise uniform. Using this and the fact that $f$ is non-negative, we can write

$$
0 \leq \mathbb{E}[f(D)] \leq \mathbb{E}[p(D)]=\mathbb{E}[p(U)] \leq \varepsilon .
$$

Hence, $\mathbb{E}[f(D)] \leq \varepsilon$. This is the method of sandwiching polynomials from Reference [3]. 
Let us write $f=g\left(\sum_{i} x_{i} / n\right)$, for $g:\{0,1 / n, \ldots, 1\} \rightarrow\{0,1\}$. We exhibit a univariate polynomial $q$ of degree $d$ such that $g(x) \leq q(x)$ for every $x$, and the expectation of $q$ under the binomial distribution is at most $\varepsilon$. The polynomial $p$ is then $q\left(\sum_{i} x_{i} / n\right)$.

Consider the continuous, piecewise linear function $s:[-1,1] \rightarrow[0,1]$ defined as follows. The function is always 0 , except at intervals of radius $a / n$ around the inputs $x$ where $g$ equals 1 , i.e., inputs $x$ such that $n x$ is congruent to $c$ modulo $m$. In those intervals, we define the following tent function: it is a piecewise linear function that goes up and down like a " $\wedge$," starting from 0 at $x-a / n$, and reaching the value of 1 linearly at $x$, then going back to 0 at $x+a / n$. We set $a=\varepsilon m / 10$.

By Jackson's theorem (see, e.g., Reference [7, Theorem 7.4] or Reference [11, Chapter 6]), for $d=$ $O\left(n \varepsilon^{-1} a^{-1}\right)=O\left(n \varepsilon^{-2} m^{-1}\right)$, there exists a univariate polynomial $q^{\prime}$ of degree $d$ that approximates $s$ with pointwise error $\varepsilon / 10$. Our polynomial $q$ is defined as $q:=q^{\prime}+\varepsilon / 10$.

It is clear that $g(x) \leq q(x)$ for every $x \in\{0,1 / n, \ldots, 1\}$. It remains to estimate $\mathbb{E}[q(U)]$.

As $q^{\prime}$ is a good approximation of $s$ we have $\mathbb{E}[q(U)] \leq 2 \varepsilon / 10+\mathbb{E}[s(U)]$. We noted in Remark 1 that the remainder modulo $m$ of $\sum U_{i}$ is $\delta$-close to uniform over $\{0,1, \ldots, m-1\}$ for $\delta=\cos (\pi / 2 m)^{n}=e^{-\Omega\left(n / m^{2}\right)}$. Now the function $s$, as a function of $\sum x_{i}$, is a periodic function with period $m$. So we have

$$
\begin{aligned}
\mathbb{E}[s(U)] & =\sum_{x=0}^{a} \operatorname{Pr}\left[\sum_{i} U_{i} \equiv x(\bmod m)\right] \cdot s(x / n) \\
& \leq(1 / m+\delta) \sum_{x=0}^{a} \frac{x}{a} \\
& \leq(1 / m+\delta) \cdot a \\
& \leq(1 / m+\delta) \cdot \varepsilon m / 10 .
\end{aligned}
$$

It follows that if $n$ is at least a large constant times $m^{2} \log m$, then we have $\mathbb{E}[s(U)] \leq 2 \varepsilon / 10$ and we conclude that $\mathbb{E}[q(U)] \leq 4 \varepsilon / 10$.

\section{TIGHT LOWER BOUND ON $k$-WISE UNIFORMITY VS. MOD $m$}

In this section, we prove Theorem 2 . Throughout the rest of this article, we will interpret $0^{0}$ as 1 . For convenience, from now on we will consider the space $\{-1,1\}^{n}$ instead of $\{0,1\}^{n}$. In particular, we will consider strings $x \in\{-1,1\}^{n}$ that satisfy $\sum_{i} x_{i} \equiv c(\bmod m)$. One can translate results stated for $\{0,1\}^{n}$ to results for $\{-1,1\}^{n}$ and vice versa using the following fact. Recall that $S_{m, c}=\{x \in$ $\left.\{0,1\}^{n}: \sum_{i} x_{i} \equiv c(\bmod m)\right\}$ and $S_{m, c}^{\prime}=\left\{y \in\{-1,1\}^{n}: \sum_{i} y_{i} \equiv c(\bmod m)\right\}$.

FACT 12. Let $x \in\{0,1\}^{n}$ and let $y \in\{-1,1\}^{n}$ be the string obtained by replacing each $x_{i}$ by $y_{i}=$ $1-2 x_{i}$. Then $x$ is in $S_{m, c}$ if and only if $y$ is in $S_{2 m, n-2 c}^{\prime}$.

Let $n$ be a positive integer. Let $X_{1}, X_{2}, \ldots, X_{n}$ be independent random variables chosen uniformly from $\{-1,1\}$. Let $B$ be the sum of all the $X_{j}$. The distribution of $B$ is a shifted binomial distribution. Note that $B$ has the same parity as $n$.

Theorem 13. Let $m$ and $n$ be positive integers and $c$ be an integer. Suppose that $m$ is odd or $n$ and c have the same parity. Let $k$ be a positive integer such that $k \leq \frac{n}{8 m^{2}}$. Then there is a probability distribution on the $c$ mod $m$ integers that matches the first $k$ moments of $B$. Furthermore, the support of the probability distribution is a subset of the support of $B$.

Theorem 2 follows from applying Fact 12 to Theorem 13.

Our goal is to come up with a distribution supported on $c \bmod m$ so that its first $k$ moments match the moments of $B$. We first start with a measure $q$ on the $c \bmod m$ integers. Here $q$ may not 
be a probability measure-its values may not sum to 1 . However, we will show that we can turn $q$ into a probability measure $p$ by a small adjustment $\Delta$ on $k+1$ appropriately chosen positive values of $q(x)$.

\subsection{Defining $C_{m, c}(x)$}

Let $m$ be a positive integer (the modulus). Let $c$ be an integer (the residue). We will assume that either $m$ is odd or $n$ and $c$ have the same parity. We will use Iverson bracket notation: $\llbracket$ true $\rrbracket=1$ and $\llbracket$ false $\rrbracket=0$. Define the comb function $C_{m, c}$ on the integers by $C_{m, c}(x)=m \llbracket x \equiv c(\bmod m) \rrbracket$ if $m$ is odd and $C_{m, c}(x)=\frac{m}{2} \llbracket x \equiv c(\bmod m) \rrbracket$ if $m$ is even.

\subsection{Defining $q(x)$}

Define the function $q$ on the integers by $q(x)=C_{m, c}(x) \operatorname{Pr}[B=x] \cdot{ }^{1}$ Note that $q$ is nonnegative. Also if $q(x) \neq 0$, then $x$ is $c(\bmod m)$ and in the support of $B$.

LEMMA 14. If $f$ is a function on the integers, then

$$
\sum_{x} q(x) f(x)=\mathbb{E}\left[C_{m, c}(B) f(B)\right] .
$$

Proof. By the definition of expected value, we have

$$
\sum_{x} q(x) f(x)=\sum_{x} \operatorname{Pr}[B=x] C_{m, c}(x) f(x)=\mathbb{E}\left[C_{m, c}(B) f(B)\right] .
$$

\subsection{Defining Lagrange Polynomials}

Let $k$ be a positive integer. Let $a_{0}, a_{1}, \ldots, a_{k}$ be $k+1$ distinct integers that are $c \bmod m, n \bmod 2$, and as close to 0 as possible. Because they are as close to 0 as possible, we have $\left|a_{j}\right| \leq(k+1) m \leq$ $2 \mathrm{~km}$. In our application, $2 \mathrm{~km}$ will be at most $n$. So each $a_{j}$ will be in the support of $B$.

Given an integer $v$ such that $0 \leq v \leq k$, define the Lagrange polynomial $L_{v}$ as follows:

$$
L_{v}(x)=\prod_{\substack{0 \leq j \leq k \\ j \neq v}}\left(x-a_{j}\right) .
$$

Note that $L_{v}\left(a_{w}\right)=0$ if and only if $v \neq w$. It is well known that $L_{0}, L_{1}, \ldots, L_{k}$ form a basis (the Lagrange basis) of the vector space of polynomials of degree at most $k$.

\subsection{Defining $\Delta(x)$}

Define the function $\Delta$ on the integers as follows. If $x$ equals $a_{v}$ (for some $v$ ), then

$$
\Delta\left(a_{v}\right)=\frac{\mathbb{E}\left[C_{m, c}(B) L_{v}(B)\right]-\mathbb{E}\left[L_{v}(B)\right]}{L_{v}\left(a_{v}\right)} .
$$

If $x \neq a_{w}$ for every $w$, then $\Delta(x)=0 .^{2}$

Lemma 15. If $f$ is a polynomial of degree at most $k$, then

$$
\sum_{x} \Delta(x) f(x)=\mathbb{E}\left[C_{m, c}(B) f(B)\right]-\mathbb{E}[f(B)] .
$$

\footnotetext{
${ }^{1}$ Alternatively, we could have defined $q$ to be the probability distribution of $B$ conditioned on $B \equiv c(\bmod m)$. While that definition would work, it would make our argument slightly more complicated.

${ }^{2}$ Why this definition of $\Delta$ ? We want $\Delta$ to be nonzero only at the $k+1$ values $a_{0}, a_{1}, \ldots, a_{k}$. We also want Lemma 15 to hold. Those two constraints force the given definition of $\Delta$.
} 
Proof. We will first prove the claim when $f$ is a Lagrange polynomial $L_{v}$. If $\Delta(x) \neq 0$, then $x$ is of the form $a_{w}$ for some $w$. But if $L_{v}\left(a_{w}\right) \neq 0$, then $v=w$. So the sum has at most one nonzero term, corresponding to $x=a_{v}$. And the equation is true in this case by the definition of $\Delta$.

We have proved the claim for Lagrange polynomials. But every polynomial of degree at most $k$ is a linear combination of the Lagrange polynomials. This completes the proof.

\subsection{Defining $p(x)$}

Define the function $p$ on the integers by $p(x)=q(x)-\Delta(x)$. Note that if $p(x) \neq 0$, then $x$ is $c$ mod $m$ and (assuming $2 \mathrm{~km} \leq n$ ) in the support of $B$.

Lemma 16. If $f$ is a polynomial of degree at most $k$, then

$$
\sum_{x} p(x) f(x)=\mathbb{E}[f(B)] .
$$

Proof. By Lemmas 14 and 15, we have

$$
\begin{aligned}
\sum_{x} p(x) f(x) & =\sum_{x} q(x) f(x)-\sum_{x} \Delta(x) f(x) \\
& =\mathbb{E}\left[C_{m, c}(B) f(B)\right]-\left(\mathbb{E}\left[C_{m, c}(B) f(B)\right]-\mathbb{E}[f(B)]\right) \\
& =\mathbb{E}[f(B)] .
\end{aligned}
$$

To show that $p$ is nonnegative, we will show that $|\Delta(x)| \leq \frac{1}{2} q(x)$. First, we bound above $|\Delta(x)|$. Then we bound below $q(x)$.

Recall that $\Delta(x)=\frac{\mathbb{E}\left[C_{m, c}(B) L_{v}(B)\right]-\mathbb{E}\left[L_{v}(B)\right]}{L_{v}\left(a_{v}\right)}$ if $x$ equals $a_{v}$ for some $v$ (and 0 otherwise). Lemmas 19 and 21 below give upper and lower bounds on the numerator and denominator, respectively.

\subsection{Bounding Numerator of $\Delta$}

We start this subsection by stating a useful lemma.

LEMMA 17. Let $r$ be an integer such that $0 \leq r \leq \frac{n}{8}$. If $0<\theta \leq \frac{\pi}{2}$, then

$$
\left|\mathbb{E}\left[B^{r} e^{i \theta B}\right]\right| \leq 2\left(\frac{8 r}{\theta}\right)^{r} e^{-\theta^{2} n / 4} .
$$

Informally, this lemma says that the $r$ th moment of $B$, after being twisted in the complex plane, is exponentially small. We defer its proof to Section 5.

Our next lemma says that $B$ is exponentially close to being uniformly distributed mod $m$ even when weighted by moments.

LEMMA 18. Let $r$ be an integer such that $0 \leq r \leq \frac{n}{8}$. Let $m$ be an integer such that $1 \leq m \leq \sqrt{n}$. Suppose that $m$ is odd or $n$ and $c$ have the same parity. Then

$$
\left|\mathbb{E}\left[B^{r} C_{m, c}(B)\right]-\mathbb{E}\left[B^{r}\right]\right| \leq 8(3 r m)^{r} e^{-2 n / m^{2}} .
$$

Proof. Let $m^{\prime}$ be $m$ if $m$ is odd and $m / 2$ if $m$ is even. Let $\alpha$ be $\pi / m^{\prime}$. For now, we will assume that $n$ and $c$ have the same parity. At the end, we will show how to adjust the proof when $n$ and $c$ have the opposite parity.

Because $B$ and $n$ have the same parity, $B-c$ is even. So we have the key identity

$$
\sum_{j=1}^{m^{\prime}-1} e^{i j \alpha(B-c)}=C_{m, c}(B)-1 .
$$


Hence, by the triangle inequality, we have

$$
\begin{aligned}
\left|\mathbb{E}\left[B^{r} C_{m, c}(B)\right]-\mathbb{E}\left[B^{r}\right]\right| & =\left|\mathbb{E}\left[B^{r} \sum_{j=1}^{m^{\prime}-1} e^{i j \alpha(B-c)}\right]\right| \\
& =\left|\sum_{j=1}^{m^{\prime}-1} e^{-i j \alpha c} \mathbb{E}\left[B^{r} e^{i j \alpha B}\right]\right| \\
& \leq \sum_{j=1}^{m^{\prime}-1}\left|\mathbb{E}\left[B^{r} e^{i j \alpha B}\right]\right| .
\end{aligned}
$$

The sum is symmetric: The terms corresponding to $j=\ell$ and $j=m^{\prime}-\ell$ are equal. So we can double its first half:

$$
\left|\mathbb{E}\left[B^{r} C_{m, c}(B)\right]-\mathbb{E}\left[B^{r}\right]\right| \leq 2 \sum_{j=1}^{\left\lfloor m^{\prime} / 2\right\rfloor}\left|\mathbb{E}\left[B^{r} e^{i j \alpha B}\right]\right| .
$$

Therefore, by Lemma 17, we have

$$
\begin{aligned}
\left|\mathbb{E}\left[B^{r} C_{m, c}(B)\right]-\mathbb{E}\left[B^{r}\right]\right| & \leq 4 \sum_{j=1}^{\left\lfloor m^{\prime} / 2\right\rfloor}\left(\frac{8 r}{j \alpha}\right)^{r} e^{-j^{2} \alpha^{2} n / 4} \\
& \leq 4\left(\frac{8 r}{\alpha}\right)^{r} \sum_{j=1}^{\left\lfloor m^{\prime} / 2\right\rfloor} e^{-j^{2} \alpha^{2} n / 4} \\
& \leq 4\left(\frac{8 r m}{\pi}\right)^{r} \sum_{j=1}^{\left\lfloor m^{\prime} / 2\right\rfloor} e^{-j^{2} \pi^{2} n /\left(4 m^{2}\right)} \\
& \leq 4(3 r m)^{r} \sum_{j=1}^{\left\lfloor m^{\prime} / 2\right\rfloor} e^{-2 j^{2} n / m^{2}} \\
& \leq 4(3 r m)^{r} \sum_{j=1}^{\left\lfloor m^{\prime} / 2\right\rfloor} e^{-2 j n / m^{2}} .
\end{aligned}
$$

The sum is a geometric series whose common ratio is less than $\frac{1}{2}$, so we can bound it by twice its first term:

$$
\left|\mathbb{E}\left[B^{r} C_{m, c}(B)\right]-\mathbb{E}\left[B^{r}\right]\right| \leq 8(3 r m)^{r} e^{-2 n / m^{2}} .
$$

The proof above assumed that $n$ and $c$ have the same parity. When $n$ and $c$ have the opposite parity, we can adjust the proof as follows. From the parity hypothesis in the theorem, we know that $m$ is odd. In particular, $m^{\prime}=m$. Because $B$ and $n$ have the same parity, $B-c$ is odd. So we have the identity

$$
\sum_{j=1}^{m^{\prime}-1}(-1)^{j} e^{i j \alpha(B-c)}=C_{m, c}(B)-1 .
$$

It is the same identity as before except for the factor of $(-1)^{j}$. We can now continue with the remainder of the proof. The factor of $(-1)^{j}$ goes away as soon as we apply the triangle inequality. Hence we obtain the same bound. 
Our final lemma of this subsection says that $B$ is exponentially close to being uniformly distributed mod $m$ even when weighted by Lagrange polynomials. It provides the upper bound on the numerator of $\Delta$ that we were seeking.

Lemma 19. Let $m$ be an integer such that $1 \leq m \leq \sqrt{n}$. Suppose that $m$ is odd or $n$ and $c$ have the same parity. Suppose that $k \leq \frac{n}{8}$. If $v$ is an integer such that $0 \leq v \leq k$, then

$$
\left|\mathbb{E}\left[L_{v}(B) C_{m, c}(B)\right]-\mathbb{E}\left[L_{v}(B)\right]\right| \leq 8(5 k m)^{k} e^{-2 n / m^{2}} .
$$

Proof. Given a subset $A$ of $\{1,2, \ldots, n\}$, define the monomial $X^{A}$ to be the product of the $X_{j}$ for which $j$ is in $A$. By expanding the product, we have

$$
L_{v}(B)=\prod_{j \neq v}\left(B-a_{j}\right)=\sum_{A}(-1)^{|A|} a^{A} B^{k-|A|},
$$

where $A$ ranges over every subset of $\{0,1, \ldots, k\}-\{v\}$. Therefore, by the triangle inequality, Lemma 18, and the binomial theorem, we have

$$
\begin{aligned}
\left|\mathbb{E}\left[L_{v}(B) C_{m, c}(B)\right]-\mathbb{E}\left[L_{v}(B)\right]\right| & =\left|\mathbb{E}\left[\left[C_{m, c}(B)-1\right] L_{v}(B)\right]\right| \\
& =\left|\mathbb{E}\left[\left[C_{m, c}(B)-1\right] \sum_{A}(-1)^{|A|} a^{A} B^{k-|A|}\right]\right| \\
& =\left|\sum_{A}(-1)^{|A|} a^{A} \mathbb{E}\left[\left[C_{m, c}(B)-1\right] B^{k-|A|}\right]\right| \\
& \leq \sum_{A}\left|a^{A} \mathbb{E}\left[\left[C_{m, c}(B)-1\right] B^{k-|A|}\right]\right| \\
& \leq \sum_{A}(2 k m)^{|A|}\left|\mathbb{E}\left[\left[C_{m, c}(B)-1\right] B^{k-|A|}\right]\right| \\
& \leq \sum_{A}(2 k m)^{|A|} \cdot 8(3(k-|A|) m)^{k-|A|} e^{-2 n / m^{2}} \\
& =8 e^{-2 n / m^{2}} \sum_{A}(2 k m)^{|A|}(3(k-|A|) m)^{k-|A|} \\
& \leq 8 e^{-2 n / m^{2}} \sum_{A}(2 k m)^{|A|}(3 k m)^{k-|A|} \\
& =8 e^{-2 n / m^{2}}(5 k m)^{k} .
\end{aligned}
$$

\subsection{Bounding Denominator of $\Delta$}

Our lower bound on $\left|L_{v}\left(a_{v}\right)\right|$ follows from the following claim.

CLAIm 20. Let $a_{0}<\cdots<a_{k}$ be $k+1$ points such that for every $j \in\{1, \ldots, k\}$ we have $a_{j}-a_{j-1} \geq$ $d$. Then for any integer $t$ such that $0 \leq t \leq k$,

$$
\prod_{j \neq t}\left|a_{t}-a_{j}\right| \geq\left(\frac{k d}{2 e}\right)^{k} .
$$

Proof. First, observe that we have $\left|a_{t}-a_{j}\right| \geq d|t-j|$. So we have

$$
\prod_{j \neq t}\left|a_{t}-a_{j}\right| \geq \prod_{j \neq t} d|t-j|=d^{k} t !(k-t) !
$$


Note that $t !(k-t) ! \geq k ! / 2^{k}$, since $\left(\begin{array}{l}k \\ t\end{array}\right) \leq 2^{k}$. Using Stirling's formula $x ! \geq(x / e)^{x}$ for positive integer $x$, we have

$$
\prod_{j \neq t}\left|a_{t}-a_{j}\right| \geq d^{k} t !(k-t) ! \geq d^{k} \frac{k !}{2^{k}} \geq d^{k}\left(\frac{k}{2 e}\right)^{k}=\left(\frac{k d}{2 e}\right)^{k} .
$$

Lemma 21. If $v$ is an integer such that $0 \leq v \leq k$, then

$$
\left|L_{v}\left(a_{v}\right)\right| \geq\left(\frac{k m}{6}\right)^{k} .
$$

Proof. Without loss of generality, assume that the $a_{j}$ are in sorted order. Then this lemma follows from Claim 20 with $d$ replaced by $m$.

\subsection{Conclude Upper Bound on $\Delta$}

LEMмA 22. Let $m$ be an integer such that $1 \leq m \leq \sqrt{n}$. Suppose that $m$ is odd or $n$ and $c$ have the same parity. Suppose that $k \leq \frac{n}{8}$. If $x$ is an integer, then

$$
|\Delta(x)| \leq 8(30)^{k} e^{-2 n / m^{2}} .
$$

Proof. If $x$ is different from $a_{v}$ for every $v$, then $\Delta(x)=0$. So we may assume that $x=a_{v}$ for some $v$. By Lemmas 19 and 21, we have

$$
\begin{aligned}
|\Delta(x)| & =\frac{\left|\mathbb{E}\left[C_{m, c}(B) L_{v}(B)\right]-\mathbb{E}\left[L_{v}(B)\right]\right|}{\left|L_{v}\left(a_{v}\right)\right|} \\
& \leq \frac{8(5 k m)^{k} e^{-2 n / m^{2}}}{(k m / 6)^{k}} \\
& =8(30)^{k} e^{-2 n / m^{2}} .
\end{aligned}
$$

\subsection{Bounding Below $q(x)$}

Lemma 23. If $a$ is an integer such that $|a| \leq n$ and $a \equiv n(\bmod 2)$, then

$$
\operatorname{Pr}[B=a] \geq 2^{-a^{2} / n} \frac{1}{2 \sqrt{n}} .
$$

Proof. The event $B=a$ is equivalent to $\frac{n+a}{2}$ of the $X_{j}$ being 1 and the other $\frac{n-a}{2}$ being -1 . Hence

$$
\operatorname{Pr}[B=a]=\frac{1}{2^{n}}\left(\begin{array}{c}
n \\
(n+a) / 2
\end{array}\right) .
$$

Using a precise form of Stirling's formula due to Robbins [29], we can bound the binomial coefficient by

$$
\left(\begin{array}{c}
n \\
(n+a) / 2
\end{array}\right) \geq 2^{H\left(\frac{1}{2}+\frac{a}{2 n}\right) n} \frac{2 \sqrt{2 \pi}}{e^{2} \sqrt{n}},
$$

where $H$ is the binary entropy function $H(x)=-x \log _{2} x-(1-x) \log _{2}(1-x)$. By using the Taylor series of $H$ at $\frac{1}{2}$, we obtain the inequality $H\left(\frac{1+\varepsilon}{2}\right) \geq 1-\varepsilon^{2}$. Plugging that inequality into our bound on the binomial coefficient gives the desired result. 


\subsection{Conclude $|\Delta(x)| \leq q(x)$}

LEMMA 24. Let $m$ and $n$ be positive integers and $c$ be an integer. Suppose that $m$ is odd or $n$ and $c$ have the same parity. Let $k$ be a positive integer such that $k \leq \frac{n}{8 m^{2}}$. If $x$ is an integer, then $|\Delta(x)| \leq \frac{1}{2} q(x)$.

PROOF. If $x$ is different from $a_{v}$ for every $v$, then $\Delta(x)=0$. So we may assume that $x=a_{v}$ for some $v$. By Lemma 22, we have

$$
|\Delta(x)| \leq 8(30)^{k} e^{-2 n / m^{2}} \leq \frac{1}{4} e^{8 k} e^{-2 n / m^{2}} \leq \frac{1}{4} e^{n / m^{2}} e^{-2 n / m^{2}}=\frac{1}{4} e^{-n / m^{2}} .
$$

By the definition of $q$ and Lemma 23, we have

$$
q(x)=C_{m, c}(x) \operatorname{Pr}[B=x] \geq \frac{m}{2} \operatorname{Pr}[B=x] \geq 2^{-x^{2} / n} \frac{m}{4 \sqrt{n}} .
$$

We know that

So

$$
|x|=\left|a_{v}\right| \leq 2 k m \leq \frac{n}{4 m} .
$$

$$
q(x) \geq 2^{-n /\left(16 m^{2}\right)} \frac{m}{4 \sqrt{n}} \geq e^{-n /\left(16 m^{2}\right)} \frac{m}{4 \sqrt{n}} .
$$

Applying the inequality $x \leq e^{x / e}$ (to $x=4 n / m^{2}$ ), we have

$$
\frac{4 n}{m^{2}} \leq e^{4 n /\left(e m^{2}\right)} \leq e^{3 n /\left(2 m^{2}\right)} .
$$

Thus,

$$
\frac{m}{4 \sqrt{n}}=\frac{1}{2}\left(\frac{4 n}{m^{2}}\right)^{-1 / 2} \geq \frac{1}{2} e^{-3 n /\left(4 m^{2}\right)}
$$

Hence

$$
q(x) \geq \frac{1}{2} e^{-3 n /\left(4 m^{2}\right)} e^{-n /\left(16 m^{2}\right)} \geq \frac{1}{2} e^{-n / m^{2}} .
$$

Comparing our bounds for $\Delta$ and $q$, we see that $|\Delta(x)| \leq \frac{1}{2} q(x)$.

\subsection{Conclude Lower Bound}

Proof of Theorem 13. Recall the function $p$ from Lemma 16 is defined as $p(x)=q(x)-\Delta(x)$. We will show that $p$ is the desired probability distribution. From the definition of $p$ and Lemma 24 , we get $p(x) \geq \frac{1}{2} q(x)$; in particular, $p$ is nonnegative. Applying Lemma 16 to the constant function 1 (the zeroth moment), we see that the sum of the $p(x)$ is 1 . In other words, $p$ is indeed a probability distribution. Applying Lemma 16 to the other monomials (namely $x, x^{2}, \ldots, x^{k}$ ), we see that $p$ matches the first $k$ moments of $B$. This completes the proof.

\section{TIGHT LOWER BOUND ON $K$-WISE UNIFORMITY VS. THRESHOLD}

In this section, we prove Theorem 6 . Like the last section, we will work with $\{-1,1\}^{n}$ and translate the results back to $\{0,1\}^{n}$ using the following fact.

FACT 25. Let $x \in\{0,1\}^{n}$ and $y \in\{-1,1\}^{n}$ be the string obtained by replacing each $x_{i}$ by $y_{i}=1-$ $2 x_{i}$. Then $\left|\sum_{i} x_{i}-n / 2\right| \leq t$ if and only if $\left|\sum_{i} y_{i}\right| \leq 2 t$.

Let $n$ be a positive integer. Let $X_{1}, X_{2}, \ldots, X_{n}$ be independent random variables chosen uniformly from $\{-1,1\}$. Let $B$ be the sum of all the $X_{j}$. The distribution of $B$ is a shifted binomial distribution. Note that $B$ has the same parity as $n$.

THeOREM 26. Let $n$ and $t$ be positive integers such that $t \leq n$. Let $k$ be a positive integer such that $k \leq \frac{t^{2}}{200 n}$. Then there is a probability distribution on the integers with absolute value at most $t$ that 
matches the first $k$ moments of B. Furthermore, the support of the probability distribution is a subset of the support of $B$.

Theorem 6 follows from applying Fact 25 to Theorem 26.

\subsection{Defining $T(x)$}

Let $m$ be an odd integer between $\frac{n}{3 t}$ and $\frac{n}{t}$. In Theorem 13, we constructed a probability distribution (call it $p^{\prime}$ ) on the $0 \bmod m$ integers that matches the first $\frac{t^{2}}{8 n}$ moments of $B$. Furthermore, the support of $p^{\prime}$ is a subset of the support of $B$. Looking at the proof, we see that $p^{\prime}(x) \geq$ $\frac{1}{2} C_{m, 0}(x) \operatorname{Pr}[B=x]$ for all $x$. Let $C^{\prime}(x)$ be $p^{\prime}(x) / \operatorname{Pr}[B=x]$ if $\operatorname{Pr}[B=x]>0$ and $C_{m, 0}(x)$ otherwise. We have $p^{\prime}(x)=C^{\prime}(x) \operatorname{Pr}[B=x]$ for all $x$. Because $p^{\prime}$ matches the first few moments of $B$, we have $\mathbb{E}\left[C^{\prime}(B) f(B)\right]=\mathbb{E}[f(B)]$ for every polynomial $f$ of degree at most $\frac{t^{2}}{8 n}$. Also, $C^{\prime}(x) \geq \frac{1}{2} C_{m, 0}(x)$ for all $x$.

Given an integer $x$, let $T(x)$ be $\llbracket|x| \leq t \rrbracket C^{\prime}(x)$.

\subsection{Defining $q(x)$}

Given an integer $x$, define $q(x)$ to be $T(x) \operatorname{Pr}[B=x]$. Note that $q$ is nonnegative. Also if $q(x) \neq 0$, then $|x| \leq t$ and $x$ is in the support of $B$.

LEMMA 27. If $f$ is a function on the integers, then

$$
\sum_{x} q(x) f(x)=\mathbb{E}[T(B) f(B)]
$$

Proof. By the definition of expected value, we have

$$
\sum_{x} q(x) f(x)=\sum_{x} \operatorname{Pr}[B=x] T(x) f(x)=\mathbb{E}[T(B) f(B)] .
$$

\subsection{Defining Lagrange Polynomials}

Let $a_{0}, a_{1}, \ldots, a_{k}$ be $k+1$ distinct integers that are $0 \bmod m, n \bmod 2$, and as close to 0 as possible. Because they are as close to 0 as possible, we have $\left|a_{j}\right| \leq(k+1) m \leq 2 \mathrm{~km}$. Because $k \leq \frac{t^{2}}{200 n}$ and $m \leq \frac{n}{t}$, we have $2 k m \leq t \leq n$. So $\left|a_{j}\right| \leq t$ and $a_{j}$ is in the support of $B$.

Given an integer $v$ such that $0 \leq v \leq k$, define the Lagrange polynomial $L_{v}$ as follows:

$$
L_{v}(x)=\prod_{\substack{0 \leq j \leq k \\ j \neq v}}\left(x-a_{j}\right) .
$$

Note that $L_{v}\left(a_{w}\right)=0$ if and only if $v \neq w$. It is well known that $L_{0}, L_{1}, \ldots, L_{k}$ form a basis (the Lagrange basis) of the vector space of polynomials of degree at most $k$.

\subsection{Defining $\Delta(x)$}

Define the function $\Delta$ on the integers as follows. If $x$ equals $a_{v}$ (for some $v$ ), then

$$
\Delta\left(a_{v}\right)=\frac{\mathbb{E}\left[T(B) L_{v}(B)\right]-\mathbb{E}\left[L_{v}(B)\right]}{L_{v}\left(a_{v}\right)} .
$$

For $x \neq a_{w}$ for any $w$, then $\Delta(x)=0$.

Lemma 28. If $f$ is a polynomial of degree at most $k$, then

$$
\sum_{x} \Delta(x) f(x)=\mathbb{E}[T(B) f(B)]-\mathbb{E}[f(B)]
$$


Proof. We will first prove the claim when $f$ is a Lagrange polynomial $L_{v}$. If $\Delta(x) \neq 0$, then $x$ is of the form $a_{w}$ for some $w$. But if $L_{v}\left(a_{w}\right) \neq 0$, then $v=w$. So the sum has at most one nonzero term, corresponding to $x=a_{v}$. And the equation is true in this case by the definition of $\Delta$.

We have proved the claim for Lagrange polynomials. But every polynomial of degree at most $k$ is a linear combination of the Lagrange polynomials. This completes the proof.

\subsection{Defining $p(x)$}

Define the function $p$ on the integers by $p(x)=q(x)-\Delta(x)$. Note that if $p(x) \neq 0$, then $|x| \leq t$ and $x$ is in the support of $B$.

LEMMA 29. If $f$ is a polynomial of degree at most $k$, then

$$
\sum_{x} p(x) f(x)=\mathbb{E}[f(B)]
$$

Proof. By Lemmas 27 and 28, we have

$$
\begin{aligned}
\sum_{x} p(x) f(x) & =\sum_{x} q(x) f(x)-\sum_{x} \Delta(x) f(x) \\
& =\mathbb{E}[T(B) f(B)]-(\mathbb{E}[T(B) f(B)]-\mathbb{E}[f(B)]) \\
& =\mathbb{E}[f(B)] .
\end{aligned}
$$

We will show that $|\Delta(x)| \leq \frac{1}{2} q(x)$. First we bound above $|\Delta(x)|$. Then we bound below $q(x)$. Recall that $\Delta(x)=\frac{\mathbb{E}\left[L_{v}(B) T(B)\right]-\mathbb{E}\left[L_{v}(B)\right]}{L_{v}\left(a_{v}\right)}$ if $x$ equals $a_{v}$ for some $v$ and 0 otherwise. Lemmas 33 and 34 below give upper and lower bounds on the numerator and denominator, respectively.

\subsection{Bounding Numerator of $\Delta$}

We start this subsection by bounding the central moments of a binomial distribution. The following fact is a special case of Khintchine's inequality [18,21]. For completeness, we provide a short, selfcontained proof. Recall that we interpret $0^{0}$ as 1 .

FACT 30. Let $d$ be a nonnegative integer. Then the $(2 d)$ th moment $\mathbb{E}\left[B^{2 d}\right]$ is at most $\frac{(2 d) !}{2^{d} d !} n^{d}$.

Proof. The odd moments of each $X_{j}$ are all 0 . The even moments of $X_{j}$ are all 1 . Let $g_{1}, g_{2}, \ldots, g_{n}$ be independent standard Gaussians (with mean zero and unit variance). The odd moments of $g_{j}$ are all 0 . If $c$ is a nonnegative integer, then the $(2 c)$ th moment of $g_{j}$ is known to be $\frac{(2 c) !}{2^{c} c !}$, the product of the positive odd integers less than $2 c$. In particular, the even moments of $g_{j}$ are all at least 1 . So the moments of $X_{j}$ are at most the corresponding moments of $g_{j}$.

Let $G$ be the sum of the $g_{j}$. When we expand $B^{2 d}$ and $G^{2 d}$, each gives a sum of $n^{2 d}$ terms. By the previous paragraph, the expectation of each term of $B^{2 d}$ is at most the expectation of the corresponding term of $G^{2 d}$. Hence the $(2 d)$ th moment of $B$ is at most the $(2 d)$ th moment of $G$. But $G$ is a Gaussian with mean zero and variance $n$. In particular, $G / \sqrt{n}$ is a standard Gaussian. So we have

$$
\mathbb{E}\left[B^{2 d}\right] \leq \mathbb{E}\left[G^{2 d}\right]=n^{d} \mathbb{E}\left[(G / \sqrt{n})^{2 d}\right]=\frac{(2 d) !}{2^{d} d !} n^{d}
$$

Corollary 31. If $d$ is a nonnegative integer, then $\mathbb{E}\left[B^{2 d}\right]$ is at most $\sqrt{2}(2 d n / e)^{d}$. 
Proof. Fact 30 says that

$$
\mathbb{E}\left[B^{2 d}\right] \leq \frac{(2 d) !}{2^{d} d !} n^{d} .
$$

Using the form of Stirling's formula due to Robbins [29], we can bound the factorials by

$$
\frac{(2 d) !}{2^{d} d !}<\sqrt{2}\left(\frac{2 d}{e}\right)^{d} \text {. }
$$

LEMMA 32. Let $n$ and $t$ be positive integers such that $t \leq n$ and $t^{2} \geq 200 n$. Let $r$ be an integer such that $0 \leq r \leq \frac{t^{2}}{9 n}$. Then

$$
\left|\mathbb{E}\left[B^{r} T(B)\right]-\mathbb{E}\left[B^{r}\right]\right| \leq 2 t^{r} e^{-t^{2} /(6 n)} .
$$

Proof. Let $s$ be a nonnegative integer such that $r+s$ is an even number between $\frac{t^{2}}{9 n}$ and $\frac{t^{2}}{8 n}$. (Because $t^{2} \geq 200 n$, there is such an $s$.) We have

$$
\llbracket|B|>t \rrbracket \leq t^{-s}|B|^{s} .^{3}
$$

Hence, by the moment-matching property of $C^{\prime}$, the definition of $T$, the triangle inequality, and Corollary 31, we have

$$
\begin{aligned}
\left|\mathbb{E}\left[B^{r}\right]-\mathbb{E}\left[B^{r} T(B)\right]\right| & =\left|\mathbb{E}\left[B^{r} C^{\prime}(B)\right]-\mathbb{E}\left[B^{r} T(B)\right]\right| \\
& =\left|\mathbb{E}\left[B^{r} C^{\prime}(B) \llbracket|B|>t \rrbracket\right]\right| \\
& \leq \mathbb{E}\left[|B|^{r} C^{\prime}(B) \llbracket|B|>t \rrbracket\right] \\
& \leq t^{-s} \mathbb{E}\left[|B|^{r+s} C^{\prime}(B)\right] \\
& =t^{-s} \mathbb{E}\left[B^{r+s} C^{\prime}(B)\right] \\
& =t^{-s} \mathbb{E}\left[B^{r+s}\right] \\
& \leq 2 t^{-s}\left(\frac{(r+s) n}{e}\right)^{(r+s) / 2} \\
& =2 t^{r}\left(\frac{(r+s) n}{e t^{2}}\right)^{(r+s) / 2} \\
& \leq 2 t^{r}\left(\frac{1}{8 e}\right)^{t^{2} /(18 n)} \\
& \leq 2 t^{r} e^{-t^{2} /(6 n)} .
\end{aligned}
$$

LemMA 33. Let $n$ and $t$ be positive integers such that $t \leq n$. Let $k$ be a positive integer such that $k \leq \frac{t^{2}}{200 n}$. If $v$ is an integer such that $0 \leq v \leq k$, then

$$
\left|\mathbb{E}\left[L_{v}(B) T(B)\right]-\mathbb{E}\left[L_{v}(B)\right]\right| \leq 2(2 t)^{k} e^{-t^{2} /(6 n)} .
$$

Proof. Recall that for a subset $A$ of $\{1,2, \ldots, n\}$, we define $X^{A}$ to be the product of the $X_{j}$ for which $j$ is in $A$.

By expanding the product, we have

$$
L_{v}(B)=\prod_{j \neq v}\left(B-a_{j}\right)=\sum_{A}(-1)^{|A|} a^{A} B^{k-|A|},
$$

\footnotetext{
${ }^{3}$ Bounding a hard threshold by a smooth function is a standard idea in concentration inequalities, used, for example, in the proofs of Markov's inequality and Chebyshev's inequality.
} 
where $A$ ranges over every subset of $\{0,1, \ldots, k\}-\{v\}$. Therefore, by the triangle inequality, Lemma 32, and the binomial theorem, we have

$$
\begin{aligned}
\left|\mathbb{E}\left[L_{v}(B) T(B)\right]-\mathbb{E}\left[L_{v}(B)\right]\right| & =\left|\mathbb{E}\left[[T(B)-1] L_{v}(B)\right]\right| \\
& =\left|\mathbb{E}\left[[T(B)-1] \sum_{A}(-1)^{|A|} a^{A} B^{k-|A|}\right]\right| \\
& =\left|\sum_{A}(-1)^{|A|} a^{A} \mathbb{E}\left[[T(B)-1] B^{k-|A|}\right]\right| \\
& \leq \sum_{A}\left|a^{A} \mathbb{E}\left[[T(B)-1] B^{k-|A|}\right]\right| \\
& \leq \sum_{A} t^{|A|}\left|\mathbb{E}\left[[T(B)-1] B^{k-|A|}\right]\right| \\
& \leq \sum_{A} t^{|A|} \cdot 2 t^{k-|A|} e^{-t^{2} /(6 n)} \\
& =2 e^{-t^{2} /(6 n)} \sum_{A} t^{k} \\
& =2 e^{-t^{2} /(6 n)}(2 t)^{k} .
\end{aligned}
$$

\subsection{Bounding Denominator of $\Delta$}

LEMmA 34. If $v$ is an integer such that $0 \leq v \leq k$, then

$$
\left|L_{v}\left(a_{v}\right)\right| \geq\left(\frac{k n}{9 t}\right)^{k} .
$$

Proof. Without loss of generality, assume that the $a_{j}$ are in sorted order. Then this lemma follows from Claim 20 (with $d$ replaced by $2 m$ ) and the bound $m \geq \frac{n}{3 t}$.

\subsection{Conclude Upper Bound on $\Delta$}

Lemma 35. Let $n$ and $t$ be positive integers such that $t \leq n$. Let $k$ be a positive integer such that $k \leq \frac{t^{2}}{200 n}$. If $x$ is an integer, then

$$
|\Delta(x)| \leq \frac{1}{50} e^{-t^{2} /(12 n)} .
$$

Proof. If $x$ is different from $a_{v}$ for every $v$, then $\Delta(x)=0$. So we may assume that $x=a_{v}$ for some $v$. By Lemmas 33 and 34, we have

$$
\begin{aligned}
|\Delta(x)| & =\frac{\left|\mathbb{E}\left[T(B) L_{v}(B)\right]-\mathbb{E}\left[L_{v}(B)\right]\right|}{\left|L_{v}\left(a_{v}\right)\right|} \\
& \leq \frac{2(2 t)^{k} e^{-t^{2} /(6 n)}}{(k n /(9 t))^{k}} \\
& =2\left(\frac{18 t^{2}}{k n}\right)^{k} e^{-t^{2} /(6 n)} \\
& \leq \frac{1}{50}\left(\frac{1800 t^{2}}{k n}\right)^{k} e^{-t^{2} /(6 n)} .
\end{aligned}
$$


The expression $\left(\frac{1800 t^{2}}{k n}\right)^{k}$ is an increasing function of $k$ on the interval $\left(0, \frac{1800 t^{2}}{e n}\right]$. Because $k \leq \frac{t^{2}}{200 n}$, we have

$$
\left(\frac{1800 t^{2}}{k n}\right)^{k} \leq(1800 \cdot 200)^{t^{2} /(200 n)} \leq e^{t^{2} /(12 n)} .
$$

Plugging this bound into our previous inequality for $|\Delta(x)|$ completes the proof.

\subsection{Conclude $|\Delta(x)| \leq q(x)$}

Lemma 36. Let $n$ and $t$ be positive integers such that $t \leq n$. Let $k$ be a positive integer such that $k \leq \frac{t^{2}}{200 n}$. If $x$ is an integer, then $|\Delta(x)| \leq \frac{1}{2} q(x)$.

Proof. If $x$ is different from $a_{v}$ for every $v$, then $\Delta(x)=0$. So we may assume that $x=a_{v}$ for some $v$. By Lemma 35, we have $|\Delta(x)| \leq \frac{1}{50} e^{-t^{2} /(12 n)}$. By the definition of $q$ and Lemma 23, we have

$$
q(x)=C^{\prime}(x) \operatorname{Pr}[B=x] \geq \frac{1}{2} C_{m, 0}(x) \operatorname{Pr}[B=x] \geq \frac{n}{6 t} \operatorname{Pr}[B=x] \geq 2^{-x^{2} / n} \frac{\sqrt{n}}{12 t} .
$$

We know that

So

$$
|x|=\left|a_{v}\right| \leq 2 k m \leq 2 \cdot \frac{t^{2}}{200 n} \cdot \frac{n}{t}=\frac{t}{100} .
$$

$$
q(x) \geq 2^{-t^{2} /(10000 n)} \frac{\sqrt{n}}{12 t} \geq e^{-t^{2} /(10000 n)} \frac{\sqrt{n}}{12 t} .
$$

Applying the inequality $x \leq e^{x / e}$ (to $x=\frac{t^{2}}{4 n}$ ), we have

$$
\frac{t^{2}}{4 n} \leq e^{t^{2} /(4 e n)} \leq e^{t^{2} /(10 n)}
$$

Thus,

Hence

$$
\frac{\sqrt{n}}{12 t}=\frac{1}{24}\left(\frac{t^{2}}{4 n}\right)^{-1 / 2} \geq \frac{1}{24} e^{-t^{2} /(20 n)}
$$

$$
q(x) \geq \frac{1}{24} e^{-t^{2} /(20 n)} e^{-t^{2} /(10000 n)} \geq \frac{1}{24} e^{-t^{2} /(12 n)} .
$$

Comparing our bounds for $\Delta$ and $q$, we see that $|\Delta(x)| \leq \frac{1}{2} q(x)$.

\subsection{Conclude Lower Bound}

Proof of Theorem 26. Recall the function $p$ from Lemma 29 is defined as $p(x)=q(x)-\Delta(x)$. We will show that $p$ is the desired probability distribution. From the definition of $p$ and Lemma 36, we get $p(x) \geq \frac{1}{2} q(x)$; in particular, $p$ is nonnegative. Applying Lemma 29 to the constant function 1 (the zeroth moment), we see that the sum of the $p(x)$ is 1 . In other words, $p$ is indeed a probability distribution. Applying Lemma 29 to the other monomials (namely $x, x^{2}, \ldots, x^{k}$ ), we see that $p$ matches the first $k$ moments of $B$. This completes the proof.

\section{TWISTED MOMENTS: PROOF OF LEMMA 17}

In this section, we will restate and then prove the following lemma that was used in Section 3.6. Recall that $X_{1}, X_{2}, \ldots, X_{n}$ are independent random variables chosen uniformly from $\{-1,1\}$, and $B$ is the sum of all the $X_{j}$. Also, we interpret $0^{0}$ as 1 .

LEMMA 17. Let $r$ be an integer such that $0 \leq r \leq \frac{n}{8}$. If $0<\theta \leq \frac{\pi}{2}$, then

$$
\left|\mathbb{E}\left[B^{r} e^{i \theta B}\right]\right| \leq 2\left(\frac{8 r}{\theta}\right)^{r} e^{-\theta^{2} n / 4} .
$$


Informally, this lemma says that the $r$ th moment of $B$, after being twisted in the complex plane, is exponentially small. Showing that this "twisted moment" is exponentially small is delicate. We have to somehow argue that lots of cancellation is taking place in the complex plane. Our approach will be to bound the delicate twisted moment by an expected value for which no cancellation is needed. That is exactly what Lemma 40 (b) below does.

Recall that for a subset $A$ of $\{1,2, \ldots, n\}$, we define the monomial $X^{A}$ to be the product of the $X_{j}$ for which $j$ is in $A$.

We will need the hyperbolic functions. Recall that $\cosh z$ is $\left(e^{z}+e^{-z}\right) / 2, \sinh z$ is $\left(e^{z}-e^{-z}\right) / 2$, $\tanh z$ is $\sinh z / \cosh z, \operatorname{coth} z$ is $\cosh z / \sinh z$, and $\operatorname{sech} z$ is $1 / \cosh z$. Also, we have the identities $\cosh (i \theta)=\cos (\theta)$ and $\sinh (i \theta)=i \sin (\theta)$.

Our first lemma will express an exponential moment in terms of hyperbolic functions.

LEMma 37. If $A$ is a subset of $\{1,2, \ldots, n\}$ and $z$ is a complex number, then

$$
\mathbb{E}\left[X^{A} e^{z B}\right]=(\sinh z)^{|A|}(\cosh z)^{n-|A|} .
$$

Proof. Independence of the $X_{j}$ makes the proof routine. Because $B$ is the sum of the $X_{j}$, we have

$$
\begin{aligned}
\mathbb{E}\left[X^{A} e^{z B}\right] & =\mathbb{E}\left[\prod_{j \in A} X_{j} \prod_{j=1}^{n} e^{z X_{j}}\right] \\
& =\mathbb{E}\left[\prod_{j \in A} X_{j} e^{z X_{j}} \prod_{j \notin A} e^{z X_{j}}\right] \\
& =\prod_{j \in A} \mathbb{E}\left[X_{j} e^{z X_{j}}\right] \prod_{j \notin A} \mathbb{E}\left[e^{z X_{j}}\right] \\
& =\prod_{j \in A} \sinh z \prod_{j \notin A} \cosh z \\
& =(\sinh z)^{|A|}(\cosh z)^{n-|A|} .
\end{aligned}
$$

Our next lemma shows how to express a monomial twisted in the complex plane as a real quantity. This lemma will play a key role in bounding twisted moments.

Lemma 38. Let $A$ be a subset of $\{1,2, \ldots, n\}$. Let $\theta$ be a real number such that $0 \leq \theta<\frac{\pi}{4}$. Then $\left|\mathbb{E}\left[X^{A} e^{i \theta B}\right]\right|=(\cos 2 \theta)^{n / 2} \mathbb{E}\left[X^{A} e^{\lambda B}\right]$, where $\lambda$ is $\frac{1}{2} \ln \frac{1+\tan \theta}{1-\tan \theta}$.

Proof. The idea is that both expected values can be expressed in terms of hyperbolic functions using Lemma 37. So all we have to do is connect the hyperbolic function of an imaginary number with the hyperbolic function of a real number.

Because $0 \leq \theta<\frac{\pi}{4}$, we have $0 \leq \tan \theta<1$, so $\lambda$ is well defined. Also $\cos 2 \theta>0$. From our choice of $\lambda$, we have

$$
\tanh \lambda=\frac{e^{2 \lambda}-1}{e^{2 \lambda}+1}=\frac{(1+\tan \theta)-(1-\tan \theta)}{(1+\tan \theta)+(1-\tan \theta)}=\tan \theta .
$$

It follows that

$$
\cosh \lambda=\frac{1}{\sqrt{1-\tanh ^{2} \lambda}}=\frac{1}{\sqrt{1-\tan ^{2} \theta}}=\frac{\cos \theta}{\sqrt{\cos ^{2} \theta-\sin ^{2} \theta}}=\frac{\cos \theta}{\sqrt{\cos 2 \theta}} .
$$

Hence

$$
\sinh \lambda=\tanh \lambda \cosh \lambda=\tan \theta \frac{\cos \theta}{\sqrt{\cos 2 \theta}}=\frac{\sin \theta}{\sqrt{\cos 2 \theta}}
$$


Therefore, applying Lemma 37 twice, we have

$$
\begin{aligned}
\left|\mathbb{E}\left[X^{A} e^{i \theta B}\right]\right| & =|\sinh i \theta|^{|A|}|\cosh i \theta|^{n-|A|} \\
& =(\sin \theta)^{|A|}(\cos \theta)^{n-|A|} \\
& =(\cos 2 \theta)^{n / 2}(\sinh \lambda)^{|A|}(\cosh \lambda)^{n-|A|} \\
& =(\cos 2 \theta)^{n / 2} \mathbb{E}\left[X^{A} e^{\lambda B}\right] .
\end{aligned}
$$

Let $r$ be a nonnegative integer. Let $f$ be a function from $\{1,2, \ldots, r\}$ to $\{1,2, \ldots, n\}$. Define $\operatorname{odd}(f)$, the odd image of $f$, to be the set of $j$ in $\{1,2, \ldots, n\}$ such that $\left|f^{-1}(j)\right|$ is odd. Note that $|\operatorname{odd}(f)| \leq r$ and $|\operatorname{odd}(f)| \leq n$.

Our next simple lemma expresses $B^{r}$ as a sum of monomials.

LEMmA 39. If $r$ is a nonnegative integer, then $B^{r}=\sum_{f} X^{\mathrm{odd}(f)}$, where the sum is over every function $f$ from $\{1, \ldots, r\}$ to $\{1, \ldots, n\}$.

Proof. The idea is to expand $B^{r}$ and exploit the constraint that each $X_{j}$ is \pm 1 . We have

$$
\begin{aligned}
B^{r} & =\sum_{f} X_{f(1)} \cdots X_{f(r)} \\
& =\sum_{f} \prod_{j=1}^{n} X_{j}^{\left|f^{-1}(j)\right|} \\
& =\sum_{f} \prod_{j=1}^{n} X_{j}^{\left|f^{-1}(j)\right| \bmod 2} \\
& =\sum_{f} \prod_{j \in \operatorname{odd}(f)} X_{j} \\
& =\sum_{f} X^{\operatorname{odd}(f)} .
\end{aligned}
$$

As mentioned at the start of this subsection, our next key lemma will bound a delicate twisted moment by a simpler real quantity. Part (a) is not surprising; part (b) is subtler. Which bound is more useful depends on the angle $\theta$. For $\theta$ small (near zero), part (b) is better, whereas for $\theta$ large $\left(\theta \geq \frac{\pi}{4}\right)$, part (b) cannot even be applied.

LEMMA 40. Let $r$ be a nonnegative integer. Let $\theta$ be a real number such that $0 \leq \theta \leq \frac{\pi}{2}$.

(a) If $r \leq n$, then $\left|\mathbb{E}\left[B^{r} e^{i \theta B}\right]\right| \leq n^{r}(\cos \theta)^{n-r}$.

(b) If $\theta<\frac{\pi}{4}$, then $\left|\mathbb{E}\left[B^{r} e^{i \theta B}\right]\right| \leq(\cos 2 \theta)^{n / 2} \mathbb{E}\left[B^{r} e^{\lambda B}\right]$, where $\lambda$ is $\frac{1}{2} \ln \frac{1+\tan \theta}{1-\tan \theta}$.

Proof. By Lemma 39 and the triangle inequality, we have

$$
\left|\mathbb{E}\left[B^{r} e^{i \theta B}\right]\right|=\left|\mathbb{E}\left[\sum_{f} X^{\operatorname{odd}(f)} e^{i \theta B}\right]\right|=\left|\sum_{f} \mathbb{E}\left[X^{\operatorname{odd}(f)} e^{i \theta B}\right]\right| \leq \sum_{f}\left|\mathbb{E}\left[X^{\operatorname{odd}(f)} e^{i \theta B}\right]\right| .
$$

We will use this inequality in both parts. 
(a) By Lemma 37, we have

$$
\begin{aligned}
\left|\mathbb{E}\left[B^{r} e^{i \theta B}\right]\right| & \leq \sum_{f}\left|\mathbb{E}\left[X^{\operatorname{odd}(f)} e^{i \theta B}\right]\right| \\
& =\sum_{f}(\sin \theta)^{|\operatorname{odd}(f)|}(\cos \theta)^{n-|\operatorname{odd}(f)|} \\
& =(\cos \theta)^{n-r} \sum_{f}(\sin \theta)^{|\operatorname{odd}(f)|}(\cos \theta)^{r-|\operatorname{odd}(f)|} \\
& \leq(\cos \theta)^{n-r} \sum_{f} 1 \\
& =n^{r}(\cos \theta)^{n-r} .
\end{aligned}
$$

(b) By Lemmas 38 and 39, we have

$$
\begin{aligned}
\left|\mathbb{E}\left[B^{r} e^{i \theta B}\right]\right| & \leq \sum_{f}\left|\mathbb{E}\left[X^{\operatorname{odd}(f)} e^{i \theta B}\right]\right| \\
& =\sum_{f}(\cos 2 \theta)^{n / 2} \mathbb{E}\left[X^{\operatorname{odd}(f)} e^{\lambda B}\right] \\
& =(\cos 2 \theta)^{n / 2} \sum_{f} \mathbb{E}\left[X^{\operatorname{odd}(f)} e^{\lambda B}\right] \\
& =(\cos 2 \theta)^{n / 2} \mathbb{E}\left[B^{r} e^{\lambda B}\right] .
\end{aligned}
$$

To apply Lemma 40(b), we need an upper bound on $\mathbb{E}\left[B^{r} e^{\lambda B}\right]$. Fortunately, we can obtain such a bound using straightforward analysis, as the next lemma shows.

LEMMA 41. If $r \geq 0$ and $\lambda>0$, then

$$
\mathbb{E}\left[|B|^{r} e^{\lambda B}\right] \leq 2\left(\frac{4 r}{\lambda}\right)^{r}\left(\cosh \frac{11}{10} \lambda\right)^{n} .
$$

Proof. First we will bound $|B|^{r}$ by an exponential. Let $a$ be $\frac{10 r}{e \lambda}$. Let us temporarily assume that $r \neq 0$. Applying the inequality $x \leq e^{x / e}$ (to $x=|B| / a$ ), we have $|B| \leq a e^{|B| /(e a)}$. Raising both sides to the $r$ th power gives $|B|^{r} \leq a^{r} e^{r|B| /(e a)}$. Plugging in the definition of $a$, we have $|B|^{r} \leq a^{r} e^{|\lambda B| / 10}$. This inequality is true for $r=0$, too.

Now we are ready to prove the desired inequality. By Lemma 37 (with $A=\emptyset$ ), we have

$$
\begin{aligned}
\mathbb{E}\left[|B|^{r} e^{\lambda B}\right] & \leq \mathbb{E}\left[|B|^{r} e^{|\lambda B|}\right] \\
& \leq a^{r} \mathbb{E}\left[e^{|\lambda B| / 10} e^{|\lambda B|}\right] \\
& =a^{r} \mathbb{E}\left[e^{11|\lambda B| / 10}\right] \\
& \leq a^{r} \mathbb{E}\left[e^{11 \lambda B / 10}+e^{-11 \lambda B / 10}\right] \\
& =2 a^{r}\left(\cosh \frac{11}{10} \lambda\right)^{n} \\
& =2\left(\frac{10 r}{e \lambda}\right)^{r}\left(\cosh \frac{11}{10} \lambda\right)^{n} \\
& \leq 2\left(\frac{4 r}{\lambda}\right)^{r}\left(\cosh \frac{11}{10} \lambda\right)^{n} .
\end{aligned}
$$

Soon we will need three standard inequalities on hyperbolic functions and trigonometric functions. We state them below without proof. 
FACT 42. If $\lambda \geq 0$, then $\tanh \lambda \leq \lambda$.

FACT 43. If $0 \leq \theta<\frac{\pi}{2}$, then $\tan \theta \geq \theta$.

FACT 44. If $0 \leq \theta \leq \frac{\pi}{2}$, then $\cos \theta \leq e^{-\theta^{2} / 2}$.

We will also need two nonstandard inequalities on hyperbolic cosine and (ordinary) cosine. We state and prove them below.

LEMMA 45. If $\lambda \geq 0$ and $c \geq 1$, then $\cosh c \lambda \leq(\cosh \lambda)^{c^{2}}$.

Proof. We will first prove that $\tanh c x \leq c \tanh x$ for every $x \geq 0$. The derivative of $\tanh$ is $\operatorname{sech}^{2}$. Because cosh is increasing on $[0, \infty)$, it follows that $\operatorname{sech}^{2} c t \leq \operatorname{sech}^{2} t$ for every $t \geq 0$. Integrating both sides (from 0 to $x$ ) gives $\frac{1}{c} \tanh c x \leq \tanh x$. Multiplying by $c$ gives $\tanh c x \leq c \tanh x$.

Next we will prove the cosh inequality. The derivative of $\ln \cosh$ is tanh. By the previous paragraph, we have $\tanh c x \leq c \tanh x$ for every $x \geq 0$. Integrating both sides (from 0 to $\lambda$ ) gives $\frac{1}{c} \ln \cosh c \lambda \leq c \ln \cosh \lambda$. Multiplying by $c$ and exponentiating gives the desired inequality.

LEMMA 46. If $\theta$ is a real number such that $\cos ^{2} \theta \geq \frac{\sqrt{5}-1}{2}$, then $\cos 2 \theta \geq \cos ^{6} \theta$.

Proof. From the hypothesis, we have

$$
\cos ^{2} \theta+\cos ^{4} \theta=\cos ^{2} \theta\left(1+\cos ^{2} \theta\right) \geq \frac{\sqrt{5}-1}{2} \cdot \frac{\sqrt{5}+1}{2}=1 .
$$

Therefore, we have

$$
\begin{aligned}
\cos ^{6} \theta & =1-\left(1-\cos ^{2} \theta\right)\left(1+\cos ^{2} \theta+\cos ^{4} \theta\right) \\
& =1-\sin ^{2} \theta\left(1+\cos ^{2} \theta+\cos ^{4} \theta\right) \\
& \leq 1-2 \sin ^{2} \theta \\
& =\cos 2 \theta .
\end{aligned}
$$

\subsection{Conclude Proof of Twisted Moments Lemma}

Finally, we have all the ingredients needed to prove our upper bound on twisted moments. We will restate the bound again and then prove it.

LEMMA 17. Let $r$ be an integer such that $0 \leq r \leq \frac{n}{8}$. If $0<\theta \leq \frac{\pi}{2}$, then

$$
\left|\mathbb{E}\left[B^{r} e^{i \theta B}\right]\right| \leq 2\left(\frac{8 r}{\theta}\right)^{r} e^{-\theta^{2} n / 4} .
$$

Proof. We will actually prove the stronger bound

$$
\left|\mathbb{E}\left[B^{r} e^{i \theta B}\right]\right| \leq 2(8 r \cot \theta)^{r}(\cos \theta)^{n / 2} .
$$

The lemma will then follow from Facts 43 and 44.

We will consider two cases: $\theta$ large (namely $\left.\cos ^{2} \theta \leq e^{-1 / e}\right)$ and $\theta$ small $\left(\cos ^{2} \theta \geq \frac{\sqrt{5}-1}{2}\right)$. Because $\frac{\sqrt{5}-1}{2}<e^{-1 / e}$, these two cases cover all possible $\theta$. For large $\theta$ we will use Lemma 40(a), whereas for small $\theta$ we will use Lemma 40(b).

Case 1: $\theta$ large $\left(\cos ^{2} \theta \leq e^{-1 / e}\right)$. Applying the inequality $x^{x} \geq e^{-1 / e}$ for $x \geq 0$ (to $x=8 r / n$ ), we have

$$
\cos ^{2} \theta \leq e^{-1 / e} \leq\left(\frac{8 r}{n}\right)^{8 r / n}
$$


Hence, by Lemma 40(a), we have

$$
\begin{aligned}
\left|\mathbb{E}\left[B^{r} e^{i \theta B}\right]\right| & \leq n^{r}(\cos \theta)^{n-r} \\
& \leq n^{r}(\cos \theta)^{3 n / 4+r} \\
& =n^{r}\left(\cos ^{2} \theta\right)^{n / 8}(\cos \theta)^{n / 2+r} \\
& \leq n^{r}\left(\frac{8 r}{n}\right)^{r}(\cos \theta)^{n / 2+r} \\
& =(8 r \cos \theta)^{r}(\cos \theta)^{n / 2} \\
& \leq(8 r \cot \theta)^{r}(\cos \theta)^{n / 2} \\
& \leq 2(8 r \cot \theta)^{r}(\cos \theta)^{n / 2} .
\end{aligned}
$$

Case 2: $\theta$ small $\left(\cos ^{2} \theta \geq \frac{\sqrt{5}-1}{2}\right)$. In particular, $\theta<\frac{\pi}{4}$. Let $\lambda$ be $\frac{1}{2} \ln \frac{1+\tan \theta}{1-\tan \theta}$. In the proof of Lemma 38, we showed that $\tanh \lambda$ is $\tan \theta$ and $\cosh \lambda$ is $\cos \theta / \sqrt{\cos 2 \theta}$. By Fact 42 , we have $\lambda \geq \tanh \lambda=\tan \theta$. Hence, by Lemmas 40 (b), 41, 45, and 46, we have

$$
\begin{aligned}
\left|\mathbb{E}\left[B^{r} e^{i \theta B}\right]\right| & \leq(\cos 2 \theta)^{n / 2} \mathbb{E}\left[B^{r} e^{\lambda B}\right] \\
& \leq(\cos 2 \theta)^{n / 2} \mathbb{E}\left[|B|^{r} e^{\lambda B}\right] \\
& \leq 2(\cos 2 \theta)^{n / 2}\left(\frac{4 r}{\lambda}\right)^{r}\left(\cosh \frac{11}{10} \lambda\right)^{n} \\
& \leq 2(\cos 2 \theta)^{n / 2}(4 r \cot \theta)^{r}\left(\cosh \frac{11}{10} \lambda\right)^{n} \\
& \leq 2(\cos 2 \theta)^{n / 2}(4 r \cot \theta)^{r}(\cosh \lambda)^{121 n / 100} \\
& \leq 2(\cos 2 \theta)^{n / 2}(4 r \cot \theta)^{r}(\cosh \lambda)^{5 n / 4} \\
& =2(4 r \cot \theta)^{r}(\cos 2 \theta)^{n / 2}\left(\frac{\cos \theta}{\sqrt{\cos 2 \theta}}\right)^{5 n / 4} \\
& =2(4 r \cot \theta)^{r} \frac{(\cos \theta)^{5 n / 4}}{(\cos 2 \theta)^{n / 8}} \\
& \leq 2(4 r \cot \theta)^{r} \frac{(\cos \theta)^{5 n / 4}}{(\cos \theta)^{3 n / 4}} \\
& =2(4 r \cot \theta)^{r}(\cos \theta)^{n / 2} \\
& \leq 2(8 r \cot \theta)^{r}(\cos \theta)^{n / 2}
\end{aligned}
$$

\section{TIGHT UPPER BOUND ON $k$-WISE UNIFORMITY VS. MOD $m$}

In this section, we prove Theorem 3. It follows from Theorem 47 below by translating the statement for $\{-1,1\}^{n}$ back to $\{0,1\}^{n}$ using Fact 12. Recall that $S_{m, c}^{\prime}=\left\{y \in\{-1,1\}^{n}: \sum_{i} y_{i} \equiv c(\bmod m)\right\}$.

THEOREM 47. Let $m$ be a positive integer and let $c$ be an integer. Let $k$ be an integer greater than or equal to 4. Suppose there is a $k$-wise uniform distribution on $\{-1,1\}^{n}$ that is supported on $S_{m, c}^{\prime}$. Then $k \leq \frac{140 n}{m^{2}}$.

We can restate the theorem using moments as follows. Let $B$ be the sum of $n$ independent random bits chosen uniformly from $\{-1,1\}$. Let $Y$ be a random variable that is always $c \bmod m$. Suppose the first $k$ moments of $Y$ match those of $B$. Then $k \leq \frac{140 n}{m^{2}}$. 
The high-level idea of the proof is as follows. We compare $\left|\mathbb{E}\left[e^{2 \pi i Y / m}\right]\right|$ and $\left|\mathbb{E}\left[e^{2 \pi i B / m}\right]\right|$. The former is 1 , because $Y$ is always $c \bmod m$. We show that the latter is less than $e^{-\Omega\left(n / m^{2}\right)}$. We then take the Taylor approximations of the exponentials. The error is given by the $k$ th term. Because the first $k$ terms are equal by the moment-matching property of $Y$, the difference of the two expectations can be bounded in terms of the $k$ th moment of $B$, which gives us an upper bound on $k$.

Proof of Theorem 47. If $m<4$, then $k \leq n \leq \frac{16 n}{m^{2}}$. So we may assume that $m \geq 4$. Let $\alpha=$ $2 \pi / m$. Because $m \geq 4$, we have $0<\alpha \leq \pi / 2$. For now, we will assume that $k$ is even. At the end, we will handle the odd case.

Because $Y$ is always $c \bmod m$, we have

$$
\left|\mathbb{E}\left[e^{i \alpha Y}\right]\right|=\left|e^{i \alpha c}\right|=1 .
$$

By Lemma 37 (with $A=\emptyset$ ) and Fact 44, we have

$$
\left|\mathbb{E}\left[e^{i \alpha B}\right]\right|=|\cos \alpha|^{n} \leq e^{-\alpha^{2} n / 2} .
$$

The error in the Taylor approximation of an exponential has the following standard bound (see for example Feller [15, Section XV.4]): for every real $\theta$ we have

$$
\left|e^{i \theta}-\sum_{j=0}^{k-1} \frac{(i \theta)^{j}}{j !}\right| \leq \frac{\theta^{k}}{k !} .
$$

Hence, by the triangle inequality, we have

$$
\left|\mathbb{E}\left[e^{i \alpha Y}\right]-\sum_{j=0}^{k-1} \frac{(i \alpha)^{j}}{j !} \mathbb{E}\left[Y^{j}\right]\right| \leq \mathbb{E}\left[\left|e^{i \alpha Y}-\sum_{j=0}^{k-1} \frac{(i \alpha Y)^{j}}{j !}\right|\right] \leq \frac{\alpha^{k}}{k !} \mathbb{E}\left[Y^{k}\right] .
$$

Similarly, we have

$$
\left|\mathbb{E}\left[e^{i \alpha B}\right]-\sum_{j=0}^{k-1} \frac{(i \alpha)^{j}}{j !} \mathbb{E}\left[B^{j}\right]\right| \leq \frac{\alpha^{k}}{k !} \mathbb{E}\left[B^{k}\right] .
$$

Because the first $k$ moments of $Y$ match those of $B$, we get a ton of cancellation:

$$
\left|\mathbb{E}\left[e^{i \alpha Y}\right]-\mathbb{E}\left[e^{i \alpha B}\right]\right| \leq \frac{\alpha^{k}}{k !} \mathbb{E}\left[Y^{k}\right]+\frac{\alpha^{k}}{k !} \mathbb{E}\left[B^{k}\right]=2 \frac{\alpha^{k}}{k !} \mathbb{E}\left[B^{k}\right] .
$$

In particular, we have

$$
1=\left|\mathbb{E}\left[e^{i \alpha Y}\right]\right| \leq\left|\mathbb{E}\left[e^{i \alpha B}\right]\right|+2 \frac{\alpha^{k}}{k !} \mathbb{E}\left[B^{k}\right] \leq e^{-\alpha^{2} n / 2}+2 \frac{\alpha^{k}}{k !} \mathbb{E}\left[B^{k}\right] .
$$

Hence, using the moment bound of Fact 30, we have

$$
1 \leq e^{-\alpha^{2} n / 2}+2 \frac{\alpha^{k}}{2^{k / 2}(k / 2) !} n^{k / 2} \leq e^{-\alpha^{2} n / 2}+\frac{2}{(k / 2) !}\left(\frac{\alpha^{2} n}{2}\right)^{k / 2} .
$$

Let $f$ be the function defined by $f(x)=e^{-x}+\frac{2}{(k / 2) !} x^{k / 2}$. Then the inequality above simplifies to $f\left(\alpha^{2} n / 2\right) \geq 1$. Note that $f(0)=1$. Also $f$ is convex on the interval $[0, \infty)$.

We claim that $f(k \sqrt{2} / 8)<1$. To prove it, we will show that the first term of $f$ is less than $\frac{1}{2}$ and the second term is at most $\frac{1}{2}$. Because $k \geq 4$, the first term indeed satisfies

$$
e^{-k \sqrt{2} / 8} \leq e^{-\sqrt{2} / 2}<\frac{1}{2}
$$


The second term is $\frac{2}{(k / 2) !}(k \sqrt{2} / 8)^{k / 2}$. If $k=4$, then this term is exactly $\frac{1}{2}$. Otherwise, $k \geq 6$, and so by Stirling's formula we have

$$
\frac{2}{(k / 2) !}\left(\frac{k \sqrt{2}}{8}\right)^{k / 2} \leq \frac{2}{(k / 2)^{k / 2} e^{-k / 2} \sqrt{\pi k}}\left(\frac{k \sqrt{2}}{8}\right)^{k / 2}=\frac{2}{\sqrt{\pi k}}\left(\frac{e \sqrt{2}}{4}\right)^{k / 2}<\frac{2}{\sqrt{\pi k}}<\frac{1}{2} .
$$

In either case, the second term is at most $\frac{1}{2}$. Hence $f(k \sqrt{2} / 8)<1$.

To summarize, $f$ is convex on $[0, \infty), f(0)=1$, and $f(k \sqrt{2} / 8)<1$. It follows that $f$ is less than 1 on the interval $(0, k \sqrt{2} / 8]$. Because $f\left(\alpha^{2} n / 2\right) \geq 1$, we have $\alpha^{2} n / 2>k \sqrt{2} / 8$. Solving for $k$ gives

$$
k<2 \sqrt{2} \alpha^{2} n=2 \sqrt{2}\left(\frac{2 \pi}{m}\right)^{2} n<\frac{112 n}{m^{2}} .
$$

So far, we assumed that $k$ is even. Now suppose that $k$ is odd. We can apply the proof above to $k-1$, which gives the bound $k-1<\frac{112 n}{m^{2}}$. Because $k \geq 5$, we have

$$
k \leq \frac{5}{4}(k-1)<\frac{5}{4} \cdot \frac{112 n}{m^{2}}=\frac{140 n}{m^{2}} .
$$

\section{TIGHT UPPER BOUND ON $k$-WISE UNIFORMITY VS. THRESHOLD}

In this section, we prove Theorem 7, which follows from Theorem 49 below by translating the statement for $\{-1,1\}^{n}$ to $\{0,1\}^{n}$ using Fact 25 .

We will show that for any $k \geq 3$, any $k$-wise uniform distribution over $\{-1,1\}^{n}$ must put nonzero probability masses on strings $x$ whose sums $\sum_{i=1}^{n} x_{i}$ are $-\Omega(\sqrt{n k})$ and $\Omega(\sqrt{n k})$ away from 0 . This result shows that the lower bound we obtain in Theorem 6 is tight. We note that this is not true for $k=2$, as when $n$ is odd, there exists a pairwise uniform distribution supported on the all -1 vector and vectors with $(n+1) / 2$ ones.

Let $X_{1}, X_{2}, \ldots, X_{n}$ be independent random variables chosen uniformly from $\{-1,1\}$. Let $B$ be the sum of all the $X_{j}$. The distribution of $B$ is a shifted binomial distribution.

First we give a lower bound on the $d$ th moment of $B$. Recall that we interpret $0^{0}$ as 1 .

CLAIM 48. Let $d$ be a nonnegative even integer such that $d \leq 2 n$. Then $\mathbb{E}\left[B^{d}\right] \geq\left(\frac{n d}{e^{2}}\right)^{d / 2}$.

Proof. Let $d=2 r$. Consider expanding $B^{2 r}$, which gives us a sum of $n^{2 r}$ terms. If for some index $i$, the variable $X_{i}$ appears an odd number of times in a term, then this term has expectation zero. So the terms with nonzero expectation are the ones in which each $X_{i}$ appears an even number of times. In particular, each such term has expectation 1. It suffices to consider the terms in which either each $X_{i}$ appears exactly twice or does not appear at all. There are $\left(\begin{array}{l}n \\ r\end{array}\right)$ ways of choosing the indices that appear twice in a term, and each term appears $(2 r) ! / 2^{r}$ number of times in the $n^{2 r}$ terms. Hence, we have

$$
\mathbb{E}\left[B^{2 r}\right] \geq\left(\begin{array}{l}
n \\
r
\end{array}\right) \frac{(2 r) !}{2^{r}} .
$$

Using the inequality $\left(\begin{array}{l}n \\ r\end{array}\right) \geq(n / r)^{r}$ and a crude form of Stirling's formula, $n ! \geq(n / e)^{n}$, we have

$$
\left(\begin{array}{l}
n \\
r
\end{array}\right) \frac{(2 r) !}{2^{r}} \geq\left(\frac{n}{r}\right)^{r}\left(\frac{2 r}{e}\right)^{2 r} \frac{1}{2^{r}}=\left(\frac{2 n r}{e^{2}}\right)^{r}
$$

proving the claim.

Theorem 49. Let $t^{+}$and $t^{-}$be two positive integers. Let $Y$ be a random variable that is supported on $\{-1,1\}^{n}$ so that $\sum_{i} Y_{i} \geq-t^{-}$and $\sum_{i} Y_{i} \leq t^{+}$. Let $k$ be a positive integer such that $k \leq n$. Suppose that both the $(2 k)$ th and $(2 k+1)$ th moments of $Y$ and $B$ are equal. Then $\min \left\{t^{-}, t^{+}\right\} \geq \sqrt{n k} / 3$. 
Remark 2. The conclusion is false when $Y$ matches only the first two moments of $B$. When $n$ is odd, there exists a pairwise uniform distribution using Hadamard matrices that is supported on the all -1 vector and vectors with $(n+1) / 2$ ones. Namely, with probability $\frac{1}{n+1}$ choose the all -1 vector and with the remaining probability $\frac{n}{n+1}$ choose a vector with $(n+1) / 2$ ones uniformly at random.

Proof. Let $p^{+}$and $p^{-}$denote $\operatorname{Pr}[Y \geq 0]$ and $\operatorname{Pr}[Y<0]$, respectively. Note that $\mathbb{E}\left[Y^{2 k+1}\right]=$ $\mathbb{E}\left[B^{2 k+1}\right]=0$. Thus,

$$
\begin{aligned}
& p^{+} \mathbb{E}\left[|Y|^{2 k+1} \mid Y \geq 0\right]-p^{-} \mathbb{E}\left[|Y|^{2 k+1} \mid Y<0\right]=\mathbb{E}\left[Y^{2 k+1}\right]=0 \\
& p^{+} \mathbb{E}\left[|Y|^{2 k+1} \mid Y \geq 0\right]+p^{-} \mathbb{E}\left[|Y|^{2 k+1} \mid Y<0\right]=\mathbb{E}\left[|Y|^{2 k+1}\right] \geq \mathbb{E}\left[Y^{2 k}\right]^{\frac{2 k+1}{2 k}},
\end{aligned}
$$

where the last inequality follows from Jensen's inequality. Summing the two relations, we have $2 p^{+} \mathbb{E}\left[|Y|^{2 k+1} \mid Y \geq 0\right] \geq \mathbb{E}\left[Y^{2 k}\right]^{2 k+1}$. Because the $(2 k)$ th moment of $Y$ is equal to the $(2 k)$ th moment of $B$, together with Claim 48 , we have $\mathbb{E}\left[Y^{2 k}\right]^{\frac{2 k+1}{2 k}}=\mathbb{E}\left[B^{2 k}\right]^{\frac{2 k+1}{2 k}} \geq\left(\frac{2 n k}{e^{2}}\right)^{\frac{2 k+1}{2}}$. Hence, there must be a point $y$ in the support of $Y$ such that $y^{2 k+1} \geq(n k / 9)^{\frac{2 k+1}{2}}$, and so $y \geq \sqrt{n k} / 3$. By symmetry, there is another point $y^{\prime}$ in the support of $Y$ such that $y^{\prime} \leq-\sqrt{n k} / 3$.

\section{ACKNOWLEDGMENTS}

We thank the anonymous referees for their helpful feedback.

\section{REFERENCES}

[1] Miklos Ajtai and Avi Wigderson. 1989. Deterministic simulation of probabilistic constant-depth circuits. Advances in Computing Research-Randomness and Computation 5 (1989), 199-223.

[2] Noga Alon, Oded Goldreich, and Yishay Mansour. 2003. Almost k-wise independence versus k-wise independence. Inf. Process. Lett. 88, 3 (2003), 107-110.

[3] Louay M. J. Bazzi. 2009. Polylogarithmic independence can fool DNF formulas. SIAM J. Comput. 38, 6 (2009), $2220-$ 2272 .

[4] Avraham Ben-Aroya, Dean Doron, and Amnon Ta-Shma. 2017. An efficient reduction from two-source to nonmalleable extractors: Achieving near-logarithmic min-entropy. In Proceedings of the 49th Annual ACM SIGACT Symposium on Theory of Computing (STOC'17). ACM, New York, 1185-1194.

[5] Ravi Boppana, Johan Håstad, Chin Ho Lee, and Emanuele Viola. 2016. Bounded independence vs. moduli. In Proceedings of the 20th International Workshop on Randomization and Computation (RANDOM'16), Leibniz International Proceedings in Informatics, Vol. 60. Schloss Dagstuhl, 24:1-24:9.

[6] Mark Braverman. 2010. Polylogarithmic independence fools $\mathrm{AC}^{0}$ circuits. J. ACM 57, 5 (2010).

[7] Neal Carothers. [n.d.]. A Short Course on Approximation Theory. Retrieved from http://fourier.math.uoc.gr/ mk/ approx1011/carothers.pdf.

[8] J. Lawrence Carter and Mark N. Wegman. 1979. Universal classes of hash functions. f. Comput. Syst. Sci. 18, 2 (1979), $143-154$.

[9] Suresh Chari, Pankaj Rohatgi, and Aravind Srinivasan. 2000. Improved algorithms via approximations of probability distributions. F. Comput. Syste. Sci. 61, 1 (2000), 81-107. DOI : https://doi.org/10.1006/jcss.1999.1695

[10] Eshan Chattopadhyay and David Zuckerman. 2016. Explicit two-source extractors and resilient functions. In Proceedings of the 48th ACM Symposium on the Theory of Computing (STOC'16). 670-683.

[11] Elliott Cheney. 1966. Introduction to Approximation Theory. McGraw-Hill, New York, NY.

[12] Ilias Diakonikolas, Parikshit Gopalan, Ragesh Jaiswal, Rocco A. Servedio, and Emanuele Viola. 2010. Bounded independence fools halfspaces. SIAM f. Comput. 39, 8 (2010), 3441-3462.

[13] Ilias Diakonikolas, Daniel Kane, and Jelani Nelson. 2010. Bounded independence fools degree-2 threshold functions. In Proceedings of the 51st IEEE Symposium on Foundations of Computer Science (FOCS'10). IEEE, 11-20.

[14] Guy Even, Oded Goldreich, Michael Luby, Noam Nisan, and Boban Velickovic. 1992. Approximations of general independent distributions. In Proceedings of the 24th ACM Symposium on the Theory of Computing (STOC'92). 10-16.

[15] William Feller. 1971. An Introduction to Probability Theory and Its Applications (2nd ed.). Vol. 2. Wiley.

[16] Parikshit Gopalan, Raghu Meka, Omer Reingold, Luca Trevisan, and Salil Vadhan. 2012. Better pseudorandom generators from milder pseudorandom restrictions. In Proceedings of the 53rd IEEE Symposium on Foundations of Computer Science (FOCS'12). 120-129. 
[17] Parikshit Gopalan, Ryan O’Donnell, Yi Wu, and David Zuckerman. 2010. Fooling functions of halfspaces under product distributions. In Proceedings of the 25th IEEE Conference on Computational Complexity (CCC'10). IEEE, 223-234.

[18] Uffe Haagerup. 1981. The best constants in the Khintchine inequality. Stud. Math. 70, 3 (1981), 231-283.

[19] Elad Haramaty, Chin Ho Lee, and Emanuele Viola. 2018. Bounded independence plus noise fools products. SIAM 7 . Comput. 47, 2 (2018), 493-523.

[20] Prahladh Harsha and Srikanth Srinivasan. 2016. On polynomial approximations to $A C^{0}$. In Proceedings of the 20th International Workshop on Randomization and Computation (RANDOM'16), Leibniz International Proceedings in Informatics, Vol. 60. Schloss Dagstuhl, 32:1-32:14.

[21] Aleksandr Khintchine. 1923. Über dyadische Brüche. Math. Zeitschr. 18, 1 (1923), 109-116. DOI : 10.1007/BF01192399

[22] Chin Ho Lee and Emanuele Viola. 2017. More on bounded independence plus noise: Pseudorandom generators for read-once polynomials. Retreived from http://www.ccs.neu.edu/home/viola/.

[23] Chin Ho Lee and Emanuele Viola. 2017. Some limitations of the sum of small-bias distributions. Theory Comput. 13, Article 16 (2017), 23 pages. DOI : 10.4086/toc.2017.v013a016

[24] Raghu Meka and David Zuckerman. 2009. Small-bias spaces for group products. In Proceedings of the 13th Workshop on Randomization and Computation (RANDOM'09), Lecture Notes in Computer Science, Vol. 5687. Springer, 658-672.

[25] Ryan O’Donnell. 2014. Analysis of Boolean Functions. Cambridge University Press.

[26] Yuval Rabani and Amir Shpilka. 2010. Explicit construction of a small epsilon-net for linear threshold functions. SIAM f. Comput. 39, 8 (2010), 3501-3520. DOI : https://doi.org/10.1137/090764190

[27] Alexander A. Razborov. 1987. Lower bounds on the dimension of schemes of bounded depth in a complete basis containing the logical addition function. Akad. Nauk SSSR. Mat. Zamet. 41, 4 (1987), 598-607.

[28] Alexander A. Razborov. 2009. A simple proof of Bazzi's theorem. ACM Trans. Comput. Theory 1, 1 (2009). https:// dl.acm.org/citation.cfm?id=1490273.

[29] Herbert Robbins. 1955. A remark on Stirling's formula. Am. Math. Month. 62, 1 (Jan. 1955), 26-29.

[30] Roman Smolensky. 1987. Algebraic methods in the theory of lower bounds for Boolean circuit complexity. In Proceedings of the 19th ACM Symposium on the Theory of Computing (STOC'87). ACM, 77-82.

[31] Avishay Tal. 2017. Tight bounds on the Fourier spectrum of $\mathrm{AC}^{0}$. In Proceedings of the 32nd Computational Complexity Conference. LIPIcs. Leibniz Int. Proc. Inform., Vol. 79. Schloss Dagstuhl. Leibniz-Zent. Inform., Wadern, 15:1-15:31.

[32] Emanuele Viola. 2017. Special topics in complexity theory. Lecture notes of the class taught at Northeastern University. Retrieved from http://www.ccs.neu.edu/home/viola/classes/spepf17.html.

[33] Emanuele Viola and Avi Wigderson. 2008. Norms, XOR lemmas, and lower bounds for polynomials and protocols. Theory Comput. 4 (2008), 137-168. DOI : 10.4086/toc.2008.v004a007

Received May 2018; revised January 2019; accepted April 2019 